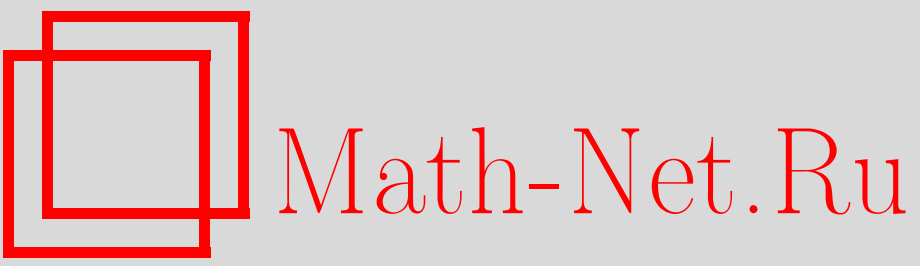

А. В. Шаповалов, Совместность случайных систем уравнений с неравновероятной выборкой двузначных неизвестных, Матем. вопр. криптогр., 2011, том 2, выпуск 4, 109-146

DOI: https://doi.org/10.4213/mvk46

Использование Общероссийского математического портала Math-Net.Ru подразумевает, что вы прочитали и согласны с пользовательским соглашением

http://www.mathnet.ru/rus/agreement

Параметры загрузки:

IP: 54.198 .67 .100

26 апреля 2023 г., 15:13:01 


\title{
Совместность случайных систем уравнений с неравновероятной выборкой двузначных неизвестных
}

\author{
А. В. Шаповалов \\ Лаборатория ТВП, Москва
}

Получено 1.XI.2010

Рассматривается случайная система уравнений относительно $n$ двузначных неизвестных, состоящая из $M=M(n)$ уравнений. Каждое уравнение содержит не более $m$ неизвестных, которые выбираются случайно и независимо. Указаны условия, при которых предельное при $M=c n^{1-1 / r}+o\left(n^{1-1 / n}\right), n \rightarrow \infty$, $2 \leq r \leq m+1, m=$ const, значение вероятности совместности убывает от 1 до 0 с ростом от 0 до $\infty$. Построен алгоритм распознавания несовместности случайной системы уравнений, имеющий при этих условиях трудоемкость $O\left(n^{1-1 / r}\right)$, $2 \leq r \leq m+1$, и асимптотически такую же вероятность распознавания несовместности как для алгоритма полного перебора.

Ключевые слова: системы дискретных уравнений, совместность, вероятностные алгоритмы, случайные гиперграфы

Satisfiability of random systems of equations with nonuniform sampling of two-valued unknowns

\section{A. V. Shapovalov}

TVP Laboratory, Moscow

Abstract. A random system of $M=M(n)$ equations with $n$ two-valued unknowns is considered. Each equation contains at most $m$ unknowns which are selected randomly. We find conditions on the structure of equations ensuring that for $M=c n^{1-1 / r}+o\left(n^{1-1 / r}\right), n \rightarrow \infty$, $2 \leq r \leq m+1, m=$ const, the probability of existence of solution decreases from 1 to 0 when $c$ increases from 0 to $\infty$. An algorithm detecting the unsolvability of a random system of equations is constructed. This algorithm has low time complexity $O\left(n^{1-1 / n}\right)$, $2 \leq r \leq m+1$. The probability of detecting the unsolvability is asymptotically the same as for the exhaustive search algorithm.

Key words: system of discrete equations, satisfiability, probabilistic algorithms, random hypergraphs

Citation: Mathematical Aspects of Cryptography, 2011, vol. 2, no. 4, pp. 109-146 (Russian). 


\section{1. Основные определения и результаты}

Пусть $\Phi_{i}=\left\{f_{i, 1}, f_{i, 2}, \ldots, f_{i, \Phi_{i} l}\right\}-$ множество функций, заданных на множестве $i$-мерных векторов, координаты которых принадлежат множеству $B$, $|B|=2, i=1, \ldots, m$. Каждая функция из $\Phi_{i}$ существенно зависит ровно от $i$ переменных и принимает значения в конечном множестве $A=\left\{a_{0}, \ldots, a_{q-1}\right\}$, $q>1$. Через $E$ обозначим декартово произведение трех множеств: $\{1, \ldots, m\}$, $\left\{1,2, \ldots, \max _{i=1, \ldots, m}\left|\Phi_{i}\right|\right\}$ и $A$. Пусть $\bar{c}=\left\{c_{i, j, a},(i, j, a) \in E\right\}$ - набор неотрицательных констант, сумма которых равна единице, и $c_{i, j, a}=0$, если $j>\left|\Phi_{i}\right|$ или $a$ не принадлежит множеству значений функции $f_{i, j}$. Для каждого $n$, $n=2,3, \ldots$, определим вектор вероятностей $\bar{p}=\bar{p}(n)=\left(p_{1}, \ldots, p_{n}\right)$, компоненты которого $p_{k}=p_{k}(n), k=1, \ldots, n$, удовлетворяют соотношениям

$$
\begin{gathered}
p_{1}+\ldots+p_{n}=1, \quad \varepsilon_{1}<n p_{k}<\varepsilon_{2}, \quad k=1, \ldots, n, \\
\lim _{n \rightarrow \infty}\left(n \sum_{k=1}^{n} p_{k}^{2}\right)=\sigma^{2},
\end{gathered}
$$

где $\varepsilon_{1}, \varepsilon_{2}, \sigma^{2}-$ некоторые положительные константы. Для краткости элементы $b_{0}$ и $b_{1}$ множества $B$ в некоторых случаях будем обозначать 0 и 1 .

Случайная система уравнений $S=S_{n, M, \bar{c}, \bar{p}}$ состоит из $M$ упорядоченных уравнений и содержит $n$ неизвестных, которые принимают значения в множестве $B$. Уравнения в случайной системе уравнений $S$ не зависят друг от друга, выбираются последовательно, вероятность появления уравнения вида

$$
f_{i, j}\left(x_{s_{1}}, \ldots, x_{s_{i}}\right)=a
$$

равна $c_{i, j, a}$ для каждого набора $(i, j, a) \in E$. Каждый индекс $s_{1}, \ldots, s_{i}$ принимает значения $1, \ldots, n$ случайно и независимо от других индексов с вероятностями $p_{1}, \ldots, p_{n}$ соответственно (выборка с возвращением), $i \in\{1, \ldots, m\}$. Индексы $i$ и $j$ в (3) означают, что функция в левой части уравнения является $j$-м элементом множества $\Phi_{i}$. Случайную систему уравнений $S$ можно определить также как систему из $M$ упорядоченных, независимых случайных уравнений вида

$$
f_{\chi_{1}, \chi_{2}}\left(x_{\xi_{1}}, \ldots, x_{\xi_{\gamma_{1}}}\right)=\chi_{3}
$$

относительно $n$ неизвестных $x_{1}, \ldots, x_{n}$, которые принимают значения в множестве $B$. Случайная величина $\left(\chi_{1}, \chi_{2}, \chi_{3}\right)$ с вероятностью $c_{i, j, a}$ принимает значение $(i, j, a)$ для каждого такого набора из $E$. Случайные независимые величины $\xi_{1}, \ldots, \xi_{\chi_{1}}$ принимают значения $1, \ldots, n$ с вероятностями $p_{1}, \ldots, p_{n}$ соответственно. 
В работе исследуется асимптотика вероятности совместности случайной системы уравнений $S$ при $n \rightarrow \infty$, когда число ее уравнений $M=M(n)$ зависит от $n$; результаты статьи [25] обобщаются на случай неравновероятного выбора неизвестных. Все асимптотические формулы приводятся при $n \rightarrow \infty$, $P\left(\lambda_{1}, \ldots, \lambda_{t}\right)$ - многомерное распределение Пуассона с независимыми компонентами и средними значениями $\lambda_{1}, \ldots, \lambda_{t}$, формула $\left(\xi_{1, n}, \ldots, \xi_{t, n}\right) \stackrel{d}{\longrightarrow} P\left(\lambda_{1}, \ldots, \lambda_{t}\right)$ означает сходимость при $n \rightarrow \infty$ последовательности распределений векторных случайных величин $\left(\xi_{1, n}, \ldots, \xi_{t, n}\right)$ к распределению $P\left(\lambda_{1}, \ldots, \lambda_{t}\right) ; \oplus-$ операция сложения в конечном поле из двух элементов; $\mathbf{b}=(b, \ldots, b)$ - вектор с $n$ одинаковыми компонентами, равными $b, b \in B ;\lfloor z\rfloor$ - целая часть числа $z,(z)_{k}=z(z-$ $1) \ldots(z-k+1)$; запись множества $\left\{x_{1}, \ldots, x_{s}\right\}$ в виде $<x_{1}, \ldots, x_{s}>$ означает, что его элементы упорядочены.

Основные используемые определения приводятся ниже и в п. 2, остальные можно найти в $[9,23,24]$. Под вероятностью некоторого свойства случайной системы уравнений (случайного гиперграфа) понимается сумма вероятностей реализаций случайной системы уравнений (случайного гиперграфа), обладающих этим свойством. Вероятность совместности $\mathbf{P}_{c}(S)$ случайной системы уравнений $S$ равна сумме вероятностей совместных реализаций этой случайной системы уравнений. Если существует функция $Q(n)$, монотонно возрастающая при $n \rightarrow \infty$ и такая, что $\mathbf{P}_{c}(S)$ стремится к единице (нулю) при $M(n) / Q(n) \rightarrow 0(M(n) / Q(n) \rightarrow \infty)$, то она называется пороговой функиией совместности случайной системы уравнений $S$. Из определения пороговой функции следует, что невозможно выделить единственную функцию с таким свойством (см., например, [30]). Таким образом, когда говорят о пороговой функции, подразумевают целый класс функций, имеющих такой же порядок роста. Вводимые ниже числовые характеристики уравнения (3) определяются при условии, что индексы его неизвестных $s_{1}, \ldots, s_{i}$ все различны. Для каждого набора $(i, j, a) \in E$ обозначим через $t_{i, j, a}^{(0)}$ и $t_{i, j, a}^{(1)}$ числа однозначно определяюшихся из уравнения (3) неизвестных, равных соответственно 0 и 1 . Под подфункцией $g_{s_{1}, \ldots, s_{t}}^{b_{1}, b_{t}}\left(y_{1}, \ldots, y_{i}\right)$ функиии $g\left(y_{1}, \ldots, y_{i}\right)$ обычно понимается функция $i-t$ переменных, получаемая из функции $g\left(y_{1}, \ldots, y_{i}\right)$ в результате фиксации переменных с номерами $s_{1}, \ldots, s_{t}$, значениями $b_{1}, \ldots, b_{t}$. Если функция $g_{s_{1}, \ldots, \ldots, s_{t}}^{b_{1}, b_{t}}\left(y_{1}, \ldots, y_{i}\right)$ является константой, то незафиксированные переменные (с номерами из $\{1, \ldots, i\} \backslash\left\{s_{1}, \ldots, s_{t}\right\}$ ) называются несущественно входящими в функцию относительно приведенной фиксации части неизвестных (см. $[6,15])$. Будем говорить, что множество $W=\left\{s_{1}, \ldots, s_{t}\right\} \subseteq\{1, \ldots, i\}$ является несовместной фиксаиией (t-фиксаиией) 
значением $b$ неизвестных уравнения (3), $b \in B$, если $g_{s_{1}, \ldots, s_{t}}^{b, \ldots,}\left(y_{1}, \ldots, y_{i}\right) \neq a$ при любых значениях $y_{k}, k \in\{1, \ldots, i\} \backslash W$, где $g=f_{i, j}, 1 \leq t \leq i, a-$ значение правой части уравнения (3). Для каждого набора $(i, j, a) \in E$ обозначим через $k_{i, j, a}^{(b, t)}$ число несовместных $t$-фиксачий значением $b$ неизвестных уравнения (3). Пусть

$$
c^{(b)}=\sum_{(i, j, a) \in E} c_{i, j, a} t_{i, j, a}^{(b)}, \quad b \in\{0,1\} ; \quad c^{(b, t)}=\sum_{(i, j, a) \in E, t \leq i} c_{i, j, a} k_{i, j, a}^{(b, t)} .
$$

Рангом несовместных фиксаций значением $b$ неизвестных уравнения (3) назовем минимальное значение $t$, при котором существует хотя бы одна несовместная фиксация значением $b$ неизвестных такого уравнения. Если $f_{i, j}(b, \ldots, b) \equiv a$, то этот ранг считается равным нулю. Обозначим через $R^{(b)}$ ранг несовместных фиксаиий значением $b$ неизвестных случайного уравнения (3a), равный минимальному значению $r$, при котором $c^{(b, r)}>0$. Если $c^{(b, r)}=0$ для каждого $r$ из множества $\{1, \ldots, m\}$, то будем считать, что $R^{(b)}=0$. Подсистема уравнений допустима в реализачиях случайной системы уравнений $S$, если она содержит не более $M$ уравнений и каждое входящее в нее уравнение имеет вид (3), причем $c_{i, j, a}>0$.

Перейдем к изложению основных результатов работы.

Теорема 1. Случайная система уравнений $S$ имеет пороговую функиию совместности $n^{1-1 / r}, 2 \leq r \leq m+1$, тогда и только тогда, когда для некоторого $b \in B$ выполняются соотношения $c^{(b)}>0, R^{(b)}=r-1$; в этом случае при

$$
M \sim c n^{1-1 / r},
$$

где с >0 — константа, справедлива формула

$$
\mathbf{P}_{c}(S) \sim e^{-\lambda}, \lambda=c^{r} c^{(b, r-1)}\left(\sigma^{2} c^{(b)}\right)^{r-1} .
$$

Теорема 2. Случайная система уравнений $S$ имеет пороговую функиию совместности п тогда и только тогда, когда $R^{(0)} R^{(1)}>0, c^{(0)}+c^{(1)}=0$.

Теорема 3. Пороговая функиия совместности случайной системы уравнений $S$ может иметь только вид п или $n^{1-1 / r}, 2 \leq r \leq m+1$.

Следуюшие условия (C1), (C2), (C3) равносильны:

(C1) случайная система уравнений $S$ имеет пороговую функиию совместности;

(C2) $R^{(0)} R^{(1)}>0$

(C3) существуют два набора $\left(i_{0}, j_{0}, a_{0}\right),\left(i_{1}, j_{1}, a_{1}\right)$, удовлетворяющие условиям

$$
f_{i_{0}, j_{0}}(0, \ldots, 0) \neq a_{0}, f_{i_{1}, j_{1}}(1, \ldots, 1) \neq a_{1}, c_{i_{0}, j_{0}, a_{0}} c_{i_{1}, j_{1}, a_{1}}>0 .
$$

Следующее утверждение вытекает из определения величины $R^{(b)}, b \in B$. 
Следствие 1. Условие $\quad R^{(b)}=0 \quad$ для $\quad b=0 \quad$ и/или $\quad b=1 \quad$ необходимо и достаточно для отсутствия пороговой функиии совместности у случайной системы уравнений $S$ и наличия у всех ее реализаций решения $\mathbf{b}$ при $b=0$ илили $b=1$.

Возможное совпадение выбираемых неизвестных для уравнения (3) не оказывает влияние на справедливость следствия 1 , так как в решении $\mathbf{b}$ все компоненты одинаковы.

В вышеприведенных утверждениях рассмотрен полный спектр возможных значений величин $R^{(0)}, R^{(1)}, c^{(0)}, c^{(1)}$. Достаточным условием справедливости соотношений (5) является существование таких $i, j$, что $c_{i, j, 0}>0$, $c_{i, j, 1}>0$. Под минимальной несовместной подсистемой системы уравнений будем понимать несовместную подсистему, которая становится совместной при исключении из нее любого уравнения. Подсистема из $l \geq 2$ уравнений соответствует гипердереву, если каждое ее уравнение не имеет совпадающих неизвестных и ее уравнения можно упорядочить так, что уравнение с номером $i=2, \ldots, l$ имеет ровно одно общее неизвестное с объединением уравнений, имеющих меньшие номера. В п. 2 приведена эквивалентная форма этого определения, раскрывающая его теоретико-графовую суть. В следующей лемме приведена полученная в статье [25] полная классификация несовместных подсистем реализаций случайной системы уравнений $S$, соответствующих гипердеревьям.

Лемма 1 ([25]). Пусть $\Phi \subseteq\left\{\Phi_{1} \cup \ldots \cup \Phi_{m}\right\}$ и $U-$ некоторое множество совместных уравнений с функциями из множества Ф. Если $U$ содержит уравнение с однозначно определяюшимся неизвестным, равным $b, b \in B$, и минимальное значение ранга несовместных фиксаиий значением $b$ неизвестных уравнений из множества $U$ равно $r-1,2 \leq r \leq m+1$, то из элементов U можно построить несовместные подсистемы уравнений, соответствующие гипердеревьям, причем любая такая минимальная подсистема состоит из г уравнений следующего вида:

(L1) функция в левой части $r$-го уравнения имеет несовместную $(r-1)$ фиксачию $W=\left\{s_{1}, \ldots, s_{r-1}\right\}$ значением $b$ неизвестных этого уравнения;

(L2) первые $r$ - 1 уравнений попарно не имеют общих неизвестных, но каждое из них имеет ровно одно общее неизвестное с $r$-м уравнением, причем это неизвестное в $k$-м уравнении однозначно определяется и равно $b$, а в $r$-м уравнении имеет номер $s_{k}, s_{k} \in W, k=1, \ldots, r-1$.

Геометрический вид описанных в лемме 1 несовместных подсистем уравнений изображен на рис. $1: r$-е уравнение $U_{r}$ (большой овал) имеет ровно по одному общему неизвестному (жирные точки) с каждым из первых $r-1$ уравнений $U_{1}, \ldots, U_{r-1}$ (маленькие овалы), которые попарно друг с другом не имеют общих неизвестных. 


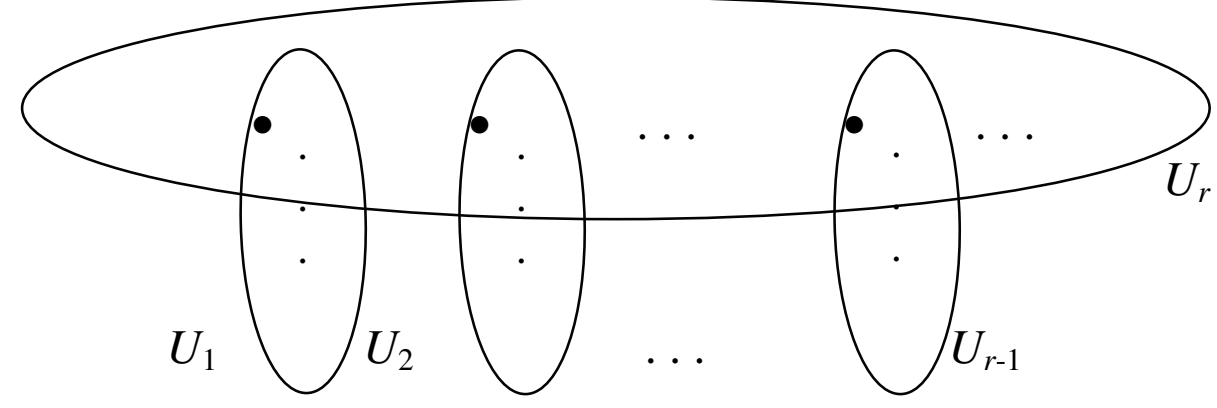

Рис. 1. Геометрический вид несовместных подсистем

Рассмотрим алгоритм $A l_{p}$ распознавания несовместности реализаций случайной системы уравнений $S$. Цель алгоритма $A l_{p}$ - найти несовместную подсистему, описываемую условиями (L1), (L2) леммы 1. Частью алгоритма $A l_{p}$ является алгоритм $A_{k l}$, определяющий наличие или отсутствие пороговой функции совместности у случайной системы уравнений $S$. При наличии пороговой функции совместности определяется ее вид, при отсутствии показывается наличие решения $\mathbf{b}$ при $b=0$ и/или $b=1$ у всех реализаций случайной системы уравнений.

\section{Алгоритм $A_{k l}$}

1. Проводится классификация уравнений вида (3) с наборами $(i, j, a) \in E$, для которых $c_{i, j, a}>0$ : выявляются уравнения с определяющимися из них неизвестными (а также номера этих неизвестных и значения неизвестных).

2. Если $c^{(0)}>0, c^{(1)}>0$, то делается вывод о том, что случайная система уравнений $S$ имеет пороговую функцию совместности $\sqrt{n}$.

3. Если $c^{(b)}>0, c^{(\bar{b})}=0$ для некоторого $b \in B$, то осуществляется перебор допустимых уравнений, выявление уравнений с несовместными фиксациями значением $b$ и определение ранга $R^{(b)}$ несовместных фиксаций значением $b$ неизвестных уравнения случайной системы $S$. В случае $R^{(b)}>0$ делается вывод о том, что случайная система уравнений $S$ имеет пороговую функцию совместности $n^{1-1 / r}, 2 \leq r \leq m+1$, где $r=R^{(b)}+1$.

4. Если $R^{(b)}=0$ и/или $R^{(\bar{b})}=0$, то делается вывод о том, что случайная система уравнений $S$ заведомо совместна и имеет решение $(b, \ldots, b)$ и/или $(\bar{b}, \ldots, \bar{b})$.

5. Если $c^{(0)}+c^{(1)}=0, R^{(0)} R^{(1)}>0$, то делается вывод о том, что случайная система уравнений $S$ имеет пороговую функцию совместности $n$. 


\section{Алгоритм $A l_{p}$}

0 . С помощью алгоритма $A_{k l}$ получается информация о значениях величин $c^{(0)}, c^{(1)}, R^{(0)}, R^{(1)}$. Если $c^{(b)}>0, R^{(b)}>0$ для $b \in B$, то определяется параметр $r=R^{(b)}+1$ пороговой функции совместности $n^{1-1 / r}$ случайной системы уравнений $S$. В остальных случаях работа алгоритма $A l_{p}$ завершается в связи с тем, что условия его эффективного применения не выполнены (в реализациях случайной системы уравнений нет несовместных подсистем, удовлетворяющих условиям (L1), (L2) леммы 1).

1. Из уравнений с однозначно определяющимися неизвестными получаются и записываются значения этих неизвестных.

2 . Если $r=2$, то $R^{(0)}=R^{(1)}=r-1=1$ и $c^{(0)}>0, c^{(1)}>0$, поэтому определяющиеся значения неизвестных могут быть 0 и 1 . Если при записи определившихся значений неизвестных системы уравнений найдется противоречие между определившимся значением неизвестного из очередного уравнения с полученным ранее значением этого неизвестного, то система уравнений несовместна; в противном случае не делается никакого вывода о ее совместности.

3. Если $r>2$, то для некоторого $b \in B$ выполнены условия $c^{(b)}>0$, $c^{(\bar{b})}=0, R^{(b)}=r-1 \geq 2$, и однозначно определяющиеся значения неизвестных могут быть равны только $b$. Перебираются уравнения с несовместными $(r-1)$-фиксациями значением $b$. Если найдется такое уравнение с фиксацией $\left\{s_{1}, \ldots, s_{r-1}\right\}$, что в нем на местах $s_{1}, \ldots, s_{r-1}$ стоят неизвестные, значения которых на первом шаге были определены (и равны $b$ ), то система уравнений несовместна; в противном случае не делается никакого вывода о ее совместности.

Под вероятностью распознавания несовместности (случайно выбираемой) реализачии случайной системы уравнений $S$ алгоритмом $A l_{p}$ будем понимать вероятность распознавания несовместности алгоритмом $A l_{p}$ (случайно выбираемой) реализации случайной системы уравнений $S$, т. е. сумму вероятностей реализации случайной системы уравнений $S$, несовместность которых этот алгоритм распознает. Максимально возможное значение этой величины - вероятность несовместности случайной системы уравнений $S$, равная $1-\mathbf{P}_{c}(S)$. Надежностью $\pi_{n}=\pi_{n}\left(A l_{p}\right)$ алгоритма $A l_{p}$ при $M>1$ будем называть вероятность распознавания несовместности (случайно выбираемой) реализации случайной системы уравнений $S$ алгоритмом $A l_{p}$ при условии несовместности этой реализации. Понятие надежности этого алгоритма имеет смысл рассматривать только для случайной системы уравнений $S$, не являющейся заведомо совместной, т. е. при $R^{(0)} R^{(1)}>0$. Легко видеть, что $0 \leq \pi_{n} \leq 1$. Алгоритм $A l_{p}$ не совершает ошибок: он может распознать несовместность несовместной реализации (хотя и не каждой), но не может признать несовместной совместную реализацию случайной системы уравнений. Поэтому при $M>1$ надежность алгоритма $A l_{p}$ равна отношению суммы ве- 
роятностей реализаций случайной системы уравнений $S$, несовместность которых алгоритм $A l_{p}$ распознает, к вероятности $1-\mathbf{P}_{c}(S)$ несовместности случайной системы уравнений $S$.

Будем говорить, что почти все несовместные реализации случайной системы уравнений обладают свойством $Q$, если вероятность свойства $Q$ при условии ее несовместности стремится к 1 при $n \rightarrow \infty$.

Теорема 4. Пусть случайная система уравнений $S$ имеет пороговую функцию совместности $n^{1-1 / r}, 2 \leq r \leq m+1$, и либо выполнено условие (4), либо $M / n^{1-1 / r} \rightarrow \infty$. Тогда почти все несовместные реализачии случайной системы уравнений $S$ содержат минимальные несовместные подсистемы из г уравнений, описанные условиями (L1), (L2) леммы 1, а надежность алгоритма Al $l_{p}$ удовлетворяет формуле $\pi_{n} \sim 1$.

При дополнительном условии $M=o\left(n^{1-1 /(r+1)}\right)$ почти все несовместные реализачии случайной системы уравнений $S$ не имеют других несовместных подсистем.

Для распознавания несовместности реализации случайной системы уравнений $S$ можно использовать алгоритм $\boldsymbol{U}$ полного перебора решений системы уравнений. Он имеет максимально возможные надежность и вероятность распознавания несовместности реализации случайной системы уравнений $S$, которые тождественно равны 1 и $1-\mathbf{P}_{c}(S)$ соответственно. В условиях теоремы 4 у алгоритма $A l_{p}$ такие же предельные значения указанных характеристик. Оценки трудоемкости алгоритмов $A l_{p}$ и $\boldsymbol{U}$ приведены в п. 4. Трудоемкость алгоритма $A l_{p}$ сравнима с простотой маркировки (просмотром) массива исходных данных (системы уравнений), поэтому в соответствии с терминологией работы [3] алгоритм $A l_{p}$ можно назвать элементарным. Для случайной системы уравнений $S$ с пороговой функцией совместности $n^{1-1 / r}, 2 \leq r \leq m+1$, при условии (4) трудоемкость алгоритма $A l_{p}$ (средняя и максимальная) является величиной порядка $n^{1-1 / r}, a$ при $R^{(0)} R^{(1)}=0$ трудоемкость конечна.

Выбор неизвестных для уравнения (3) осуществляется по схеме с возвращением. Если среди выбранных для него неизвестных окажутся совпадающие, то его функция будет иметь менее $i$ существенных переменных, а в некоторых случаях уравнения могут оказаться несовместными. Так, например, булево уравнение $x_{s_{1}} \oplus x_{s_{2}}=1$ несовместно при выборе для него двух одинаковых неизвестных. В лемме 1 статьи [28] показано, что при $M=o(n)$ почти все реализации случайной системы уравнений $S$ не имеют уравнений с совпадающими неизвестными. Результаты работы, в которых не выполнено условие $M=o(n)$, получены с помощью леммы 3, в доказательстве которой учтено возможное совпадение неизвестных в уравнениях. 
Сформулированные результаты верны и для бесповторной выборки неизвестных, когда вероятность выбора различных неизвестных $x_{s_{1}}, \ldots, x_{s_{i}}$ для уравнения (3) определяется формулой

$$
p_{s_{1}} \frac{p_{s_{2}}}{\left(1-p_{s_{1}}\right)} \frac{p_{s_{3}}}{\left(1-p_{s_{1}}-p_{s_{2}}\right)} \ldots \frac{p_{s_{i}}}{\left(1-p_{s_{1}}-\ldots-p_{s_{i-1}}\right)} .
$$

Следствие 2. Утверждения теорем 1-4 и следствия 1 справедливы при бесповторной выборке неизвестных.

Как и в [24], [28], утверждения работы справедливы для случайных систем с известными в вариантах правыми частями уравнений, в частности, для случайных систем неравенств над конечным упорядоченным множеством, а также для случайных систем уравнений с векторными функциями (см. [24]).

Условия совместности близких к $S$ случайных систем линейных и нелинейных уравнений исследовались Г. В. Балакиным, В. Ф. Колчиным, В. А. Копытцевым, В. Е. Степановым, В. И. Хохловым, автором данной работы. Публикации по случайным системам линейных булевых уравнений появились и в зарубежной литературе (см., например, [31], [32]). Примеры случайных систем уравнений с пороговой функцией совместности $\sqrt{n}$ при равновероятном бесповторном выборе неизвестных рассматривались Г. В. Балакиным (система булевых уравнений вида $x_{i} \vee \bar{x}_{j}=a$, система псевдобулевых уравнений вида $x_{1}+\ldots+x_{m}=a, a=0,1, \ldots, m$, см. [1], [5], [29]), В. А. Копытцевым (система псевдобулевых уравнений вида $x_{s_{1}} \vee \bar{x}_{s_{2}}+\ldots+x_{s_{2 d-1}} \vee \bar{x}_{s_{2 d}}=a, d \geq 1$, $a=0,1, \ldots, d)$. Примеры случайных систем уравнений с пороговой функцией совместности вида $n^{2 / 3}$ при равновероятном бесповторном выборе неизвестных рассматривались Г. В. Балакиным (система булевых уравнения вида $x_{i} \vee x_{j}=a$, см. [1]) и В. А. Копытцевым (система псевдобулевых уравнений вида $\left.x_{s_{1}} \vee x_{s_{2}}+\ldots+x_{s_{2 d-1}} \vee x_{s_{2 d}}=a, d \geq 1, a=0,1, \ldots, d\right)$. В работе [29] изучались примеры случайной системы уравнений $S$ при $B=\{-1,1\}$ и равновероятном бесповторном выборе неизвестных. Г. В. Балакиным, В. А. Копытцевым рассматривались также алгоритмы распознавания несовместности реализаций случайных систем уравнений с вышеприведенными псевдобулевыми функциями. В статье [2] развивается вероятностный подход к решению систем уравнений с целочисленными неизвестными, включающий нахождение апостериорных распределений значений неизвестных и построение окрестностей для каждого оцениваемого неизвестного. В работе [7] предлагается решать системы уравнений с небольшим числом неизвестных в каждом уравнении методом последовательного подбора неизвестных после приведения матрицы инциденций неизвестных и уравнений к специальному блочному виду. 
Понятие пороговой функции совместности случайной системы уравнений введено в работе [23], где показана ее связь с пороговой функцией для наличия подграфов заданного вида в случайных графах и гиперграфах (см. также [24-28], [30], [33], [34]). В [23], [25] построены примеры случайных систем уравнений с пороговыми функциями совместности вида $n^{1-1 / r}$, $r=2, \ldots, m+1$. Примерами случайных систем уравнений с пороговой функцией совместности $n$ являются случайные системы линейных уравнений над конечным полем и кольцом вычетов (см. [8], [10-14], [23], [26], [27], [31], [32], [34]). Результат [16] В. А. Копытцева о пороговом эффекте для среднего значения числа решений случайных систем весьма общего вида с равновероятным бесповторным выбором неизвестных показывает, что пороговая функция совместности, если она вообще существует, не может быть величиной большего порядка, чем $n$ (см. [24], [25]). Понятие однозначно определяющихся из уравнения переменных тесно связано с теорией запретов и полузапретов (см., например, [17], [24]). Результаты и обзоры по данной тематике можно найти в [1-8], [10-16], [18], [19], [22-34].

\section{2. Геометрические свойства случайной системы уравнений}

Для изучения геометрических свойств реализаций случайной системы уравнений $S$ каждой ее реализации сопоставляется гиперграф (см. [10-12], [23-28], [34]). При этом неизвестному $x_{j}$ соответствует вершина $v_{j}$, а каждое уравнение системы однозначно определяет соответствующее ему ребро гиперграфа: ребро состоит из упорядоченного набора вершин, соответствующих существенным переменным функции в уравнении. Порядок на множестве ребер гиперграфа определяется порядком на множестве уравнений соответствующей ему системы уравнений. Порядок на множестве вершин ребра определяется порядком на множестве неизвестных уравнений. Каждое ребро имеет метку, выбираемую из множества $E$ : метка ребра равна $(i, j, a)$ тогда и только тогда, когда в левой части соответствующего ему уравнения стоит функция $f_{i, j}$, а в правой части стоит элемент $a$. Установленное соответствие между системами уравнений и гиперграфами с отношениями порядка на множестве ребер и множествах вершин на каждом ребре является взаимно однозначным. Случайной системе уравнений $S$ сопоставляется случайный гиперграф $M G(S)$, реализациями которого являются гиперграфы с множеством вершин $\left\{v_{1}, \ldots, v_{n}\right\}$ и $M$ упорядоченными ребрами. Вероятности реализаций случайного гиперграфа $M G(S)$ равны вероятностям соответствующих им реализаций случайной системы уравнений $S$.

Можно дать следующие эквивалентные определения случайной системы уравнений $S$ и случайного гиперграфа $M G(S)$. На первом этапе последо- 
вательно и независимо с вероятностями $c_{i}=\sum_{(j, a)} c_{i, j, a}$ выбираются числа $i$ существенных неизвестных в $M$ уравнениях (числа вершин в $M$ ребрах), $i=1, \ldots, m, c_{1}+\ldots+c_{m}=1$. Затем для каждого уравнения, зависящего от $i$ неизвестных (ребра из $i$ вершин), независимо от других уравнений (ребер) по схеме с возвращением выбирается упорядоченный набор $i$ неизвестных (вершин), вероятность выбора неизвестного $x_{k}$ (вершины $v_{k}$ ) равна $p_{k}$, $k=1, \ldots, n$. На втором этапе для каждого уравнения, зависящего от $i$ неизвестных (ребра из $i$ вершин), при $c_{\mathrm{i}}>0$ независимо от других уравнений (ребер) с вероятностью $c_{i, j, a} / c_{i}$ выбираются функция $f_{i, j}$ из $\Phi_{i}$ и правая часть $a$ из множества $A$ (вторая и третья компоненты метки ребра).

Свойства геометрической структуры гиперграфа определяются величинами $c_{1}, \ldots, c_{m}$ и $p_{1}, \ldots, p_{n}$. Если во втором определении случайного гиперграфа $M G(S)$ остановиться после первого этапа, то получится случайный гиперграф $M G_{n, M, \bar{c}, \bar{p}}=M G_{n, \bar{c}, \bar{p}}$, свойства которого будут изучены в необходимом объеме в этом разделе работы.

Ребро гиперграфа простое, если все его вершины различны, и особое в противном случае. Рангом ребра будем называть число вершин гиперграфа, инцидентных этому ребру. Гиперграф называется ا-графом, если он имеет $l_{i}$ ребер ранга $i, i=1, \ldots, m$, где $\mathbf{I}=\left(l_{1}, \ldots, l_{m}\right), l=l_{1}+\ldots+l_{m} ; \mathbf{l}$-ииклом (при $l_{1}=0$ ) называется ا-граф, имеющий единственный цикл длины $l$ (см. [23]); в данной работе рассматриваются $\mathbf{l}$-графы только при $l_{1}=0$. Простым цик-

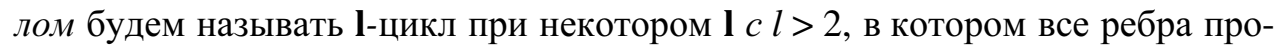
стые и каждая пара инцидентных ребер имеет ровно одну общую вершину. Гипердеревом называется связный гиперграф без циклов.

В работе используется следующее определение изоморфизма гиперграфов. Гиперграфы $H=(V, Y)$ и $H^{*}=\left(V^{*}, Y^{*}\right)$ изоморфны, если существуют отображения $\sigma_{1}$ и $\sigma_{2}$, взаимно однозначно отображающие множества вершин и множества ребер: $\sigma_{1}: V \Leftrightarrow V^{*}, \sigma_{2}: Y \Leftrightarrow Y^{*}$, с сохранением инцидентности вершин ребрам, номеров вершин в ребрах, меток ребер. Автоморфизмом гиперграфа называется изоморфное отображение этого гиперграфа на себя. Изоморфный гиперграфу $H$ подграф гиперграфа $F$ будем называть $H$-графом. В гиперграфе $H$ подграф $H^{*}$ является изолированныл, если ребра гиперграфа $H^{*}$ могут быть инцидентны только его же ребрам.

Произведением вероятностей меток подграфа $H$ реализации случайного гиперграфа $M G(S)\left(M G_{n, \bar{c}, \bar{p}}\right)$ будем называть величину $\phi(H)=$ $=c_{r_{1}, j_{1}, a_{1}} \ldots c_{r_{1}, j_{l}, a_{l}}\left(\phi(H)=c_{r_{1}} \ldots c_{r_{l}}\right)$. Здесь $r_{1}, \ldots, r_{l}$ - ранги ребер $H$, a $\left(r_{s}, j_{s}, a_{s}\right)-$ метка $s$-го ребра $H$ в $M G(S), s=1, \ldots, l$. 
Теорема 5. При $M=o(n)$ почти все реализачии случайных гиперграфов $M G_{n, \bar{c}, \bar{p}}$ и $M G(S)$ не имеют циклов.

Теорема 6. При $M=o\left(n^{1-1 / l}\right)$ почти все реализачии случайных гиперграфов $M G_{n, \bar{c}, \bar{p}}$ и $M G(S)$ не имеют гипердеревьев с l ребрами.

Рассмотрим неизоморфные гипердеревья с $l \geq 2$ ребрами $H_{1}, \ldots, H_{t}$, соответствующие несовместным подсистемам из $l=r$ уравнений, описываемым условиями (L1), (L2) леммы 1 (см. рис. 1). Упорядочим ребра в этих гиперграфах так, чтобы $l$-е ребро имело ровно по одной общей вершине с каждым из остальных $l-1$ ребер, которые попарно друг с другом не имеют общих вершин. Пусть ребра гиперграфа $H_{k}$ в соответствии с их порядком имеют метки $\left(r_{1}^{(k)}, j_{1}^{(k)}, a_{1}^{(k)}\right), \ldots, \quad\left(r_{l}^{(k)}, j_{l}^{(k)}, a_{l}^{(k)}\right), k=1, \ldots, t ;$ пусть $\phi^{(k)}=\prod_{s=1}^{l} c_{r_{s}^{(k)}, j_{s}^{(k)}, a_{s}^{(k)}}>0-$ произведение вероятностей меток ребер гиперграфа $H_{k}$. Через $\tau_{k}=\tau_{k, n}$ и $\tau_{k}^{*}=\tau_{k, n}^{*}$ обозначим числа $H_{k}$-графов и изолированных $H_{k}$-графов в случайном гиперграфе $M G(S), k=1, \ldots, t$.

Теорема 7. Если $M \sim c n^{1-1 / l}, c>0$, то

$$
\left(\tau_{1}, \ldots, \tau_{t}\right) \stackrel{d}{\longrightarrow} P\left(\lambda_{1}, \ldots, \lambda_{t}\right), \tau_{1}+\ldots+\tau_{t} \stackrel{d}{\longrightarrow} P\left(\lambda_{1}+\ldots+\lambda_{t}\right),
$$

где $\lambda_{k}=c^{l} \phi^{(k)} \sigma^{2(l-1)}, k=1, \ldots, t$.

Следствие 3. Утверждения теорем 5-7 справедливы при бесповторной выборке вершин в ребрах случайных гиперграфов.

Доказательство теоремы 5. Гиперграф с циклом содержит либо особое ребро, либо два (простых) ребра с двумя или более общими вершинами, либо простой цикл. В лемме 1 статьи [28] показано, что при $M=o(n)$ почти все реализации случайного гиперграфа $M G(S)$ (следовательно - и $M G_{n, \bar{c}, \bar{p}}$ ) не имеют особых ребер и пар ребер с двумя и более общими вершинами (в том числе - циклов длины два). Остается показать, что почти все реализации случайного гиперграфа не имеют простых циклов.

Пусть $C_{l}=C_{l}(n)$ - число простых циклов длины $l, C_{\mathrm{l}}(n)$ - число простых l-циклов, $C=C(n)$ - число простых циклов в случайном гиперграфе $M G_{n, \bar{c}, \bar{p}}$, имеющих длину три и более. Тогда $C=C_{3}+\ldots+C_{n}, C_{l}=\sum_{\text {l: } l_{2}+\ldots+l_{m}=l} C_{1}$. Через $\mathbf{i}_{1}=<i_{1,1}, \ldots, i_{1, r_{1}}>, \ldots, \mathbf{i}_{l}=<i_{l, 1}, \ldots, i_{l, r_{i}}>$ будем обозначать набор упорядоченных множеств, состоящих из $r_{1}, r_{2}, \ldots, r_{l}$ элементов соответственно, выбираемых из множества $\{1, \ldots, n\}$, причем в $\mathbf{i}_{s}$ все элементы различны, $s=1, \ldots, l$.

Пусть $l$ ребер, состоящих из вершин с индексами из множеств $\mathbf{i}_{1}, \ldots, \mathbf{i}_{l}$, образуют $\mathbf{l}$-цикл, где $\mathbf{l}=\left(l_{2}, \ldots, l_{m}\right)$, причем $\left|\mathbf{i}_{t} \cap \mathbf{i}_{t+1}\right|=1, t=1, \ldots, l-1$, 
и $\left|\mathbf{i}_{1} \bigcap \mathbf{i}_{l}\right|=1$. Для каждых таких $l$ множеств и различных чисел $N_{1}, \ldots, N_{l}$, выбираемых из множества $\{1, \ldots, M\}$, обозначим через $I\left(\mathbf{i}_{1}, \ldots, \mathbf{i}_{l}, N_{1}, \ldots, N_{l}\right)$ случайную величину, равную индикатору события, заключающегося в том, что в случайном гиперграфе $M G_{n, \bar{c}, \bar{p}}$ имеется l-цикл, в котором 1-е, ..., $l$-е ребра состоят из упорядоченных вершин, индексы которых образуют множества $\mathbf{i}_{1}, \ldots, \mathbf{i}_{l}$ соответственно, и имеют номера $N_{1}, \ldots, N_{l}$ соответственно. Каждому набору из $l$ ребер, состоящих из вершин с индексами из множеств $\mathbf{i}_{1}, \ldots, \mathbf{i}_{l}$, и номерам $\left\langle N_{1}, \ldots, N_{l}>\right.$ соответствуют ровно $2 l$ наборов множеств и номеров, которые определяют одно и тоже событие - наличие простого цикла из $l$ ребер с учетом их относительного порядка и номеров ребер в реализациях случайного гиперграфа. Первые $l$ наборов множеств получаются в результате сдвига на $j, j=0,1, \ldots, l-1$, по циклу длины $l$ всех множеств в наборе $\mathbf{i}_{1}, \ldots, \mathbf{i}_{l}$ : множество $\mathbf{i}_{s}$ получает номер $s+j$ при $s+j<l+1$ и номер $s+j-l$ при $s+j>l, s=1, \ldots, l$. Остальные $l$ наборов множеств получаются из $l$ вышеописанных наборов одновременной сменой номеров, при которой множество $\mathbf{i}_{s}$ с номером $s$ получает номер $l-s+1, s=1, \ldots, l$.

Если гиперграф, образованный $l$ ребрами, состоящими из вершин с индексами из множеств $\mathbf{i}_{1}, \ldots, \mathbf{i}_{l}$, является $\mathbf{l}$-циклом, то

$$
\mathbf{E} I\left(\mathbf{i}_{1}, \ldots, \mathbf{i}_{l} ; N_{1}, \ldots, N_{l}\right)=c_{r_{1}} \ldots c_{r_{i}} \prod_{s=1}^{l} \prod_{i \in \mathbf{i}_{s}} p_{i},
$$

где $c_{r_{1}} \ldots c_{r_{i}}-$ вероятность того, что $l$ ребер случайного гиперграфа с номерами $N_{1}, \ldots, N_{l}$ имеют ранги $r_{1}, \ldots, r_{l}, \prod_{s=1}^{l} \prod_{i \in \mathbf{i}_{s}} p_{i}$ - вероятность того, что в случайном гиперграфе ребра с номерами $N_{1}, \ldots, N_{l}$ состоят из упорядоченных вершин, индексы которых образуют множества $\mathbf{i}_{1}, \ldots, \mathbf{i}_{l}$ соответственно. Тогда число l-циклов можно представить в следующем виде:

$$
C_{1}=\frac{1}{2 l} \sum_{\mathbf{i}_{1}, \ldots, \mathbf{i}_{l}} \sum_{\left\langle N_{1}, \ldots, N_{l}\right\rangle} I\left(\mathbf{i}_{1}, \ldots, \mathbf{i}_{l} ; N_{1}, \ldots, N_{l}\right) .
$$

В этой формуле внутреннее суммирование осуществляется по упорядоченным наборам попарно различных номеров ребер $\left\langle N_{1}, \ldots, N_{l}\right\rangle$, выбираемых из множества $\{1, \ldots, M\}$; в сумме по $\mathbf{i}_{1}, \ldots, \mathbf{i}_{l}$ перебираются множества индексов, для которых $l$ ребер, состоящих из вершин с индексами из этих множеств, образуют простой цикл; множитель $\frac{1}{2 l}$ обусловлен наличием $2 l$ наборов множеств $\mathbf{i}_{1}, \ldots, \mathbf{i}_{l}$ и номеров ребер $\left\langle N_{1}, \ldots, N_{l}\right\rangle$, соответствующих одному и тому же простому циклу из $l$ ребер. В сумме по $\left\langle N_{1}, \ldots, N_{l}>\right.$ для каждого набора $\mathbf{i}_{1}, \ldots, \mathbf{i}_{l}$ имеется $(M)_{l}$ различных вариантов выбора $l$ упоря- 
доченных номеров ребер для этих $l$ ребер, а все слагаемые этой суммы одинаковы и определяются формулой (6), поэтому

$$
\mathbf{E} C_{1}=\frac{(M)_{l}}{2 l} c_{r_{1}} \ldots c_{r_{i}} \sum_{\mathbf{i}_{1}, \ldots, \mathbf{i}_{l}} \prod_{s=1}^{l} \prod_{i \in \mathbf{i}_{s}} p_{i} .
$$

Наборы индексов $\mathbf{i}_{1}, \ldots, \mathbf{i}_{l}$ удовлетворяют условиям $s_{t, t+1}=\mathbf{i}_{t} \cap \mathbf{i}_{t+1}, t=1, \ldots$, $l-1$, и $s_{l, 1}=\mathbf{i}_{1} \cap \mathbf{i}_{l}$, причем элементы $s_{1,2}, s_{2,3}, \ldots, s_{l, 1}$ попарно различны. Имеется $\left(r_{1}\right)_{2}\left(r_{2}\right)_{2} \ldots\left(r_{l}\right)_{2}$ вариантов выбора номеров элементов $s_{1,2}, s_{2,3}, \ldots, s_{l, 1}$ в наборах $\mathbf{i}_{1}, \ldots, \mathbf{i}_{l}$, а в слагаемых суммы по $\mathbf{i}_{1}, \ldots, \mathbf{i}_{l}$ вероятности с индексами $s_{1,2}, s_{2,3}, \ldots, s_{l, 1}$ встречаются дважды, с остальными индексами - по одному разу. Сумму по наборам $\mathbf{i}_{1}, \ldots, \mathbf{i}_{l}$ можно оценить сверху суммой по индексам $i_{1,1}, \ldots, i_{1, r_{1}}, \ldots, i_{l, 1}, \ldots, i_{l, r_{i}}$, расширив до множества $\{1, \ldots, n\}$ диапазон суммирования по каждому индексу:

$$
\sum_{\mathbf{i}_{1}, \ldots, \mathbf{i}_{l}} \prod_{s=1}^{l} \prod_{i \in \mathbf{i}_{s}} p_{i} \leq\left(r_{1}\right)_{2} \ldots\left(r_{l}\right)_{2} \sum_{s_{1,2}=1}^{n} p_{s_{1,2}}^{2} \ldots \sum_{s_{l-1, l}=1}^{n} p_{s_{l-1, l}}^{2} \sum_{s_{l, 1}=1}^{n} p_{s_{l, 1}}^{2} .
$$

Здесь использована формула (1). Отсюда следует, что

$$
\mathbf{E} C_{\mathbf{l}} \leq \frac{(M)_{l}}{2 l} c_{r_{1}} \ldots c_{r_{l}}\left(r_{1}\right)_{2} \ldots\left(r_{l}\right)_{2} \sum_{s_{1,2}=1}^{n} p_{s_{1,2}}^{2} \ldots \sum_{s_{l-1, l}=1}^{n} p_{s_{l-1, l}}^{2} \sum_{s_{l, 1}=1}^{n} p_{s_{l, 1}}^{2}
$$

и

$$
\mathbf{E} C_{l}=\sum_{\mathbf{l}} \mathbf{E} C_{\mathbf{l}} \leq \frac{(M)_{l}}{2 l} \sum_{r_{1}=2}^{m} c_{r_{1}}\left(r_{1}\right)_{2} \ldots \sum_{\eta_{l}=2}^{m} c_{\eta_{l}}\left(r_{l}\right)_{2} \sum_{s_{1,2}=1}^{n} p_{s_{1,2}}^{2} \ldots \sum_{s_{l, 1}=1}^{n} p_{s_{l, 1}}^{2} \leq \frac{D_{M, n}^{l}}{2 l},
$$

где $m$ - максимальное число неизвестных в уравнениях случайной системы уравнений $S$ (вершин в ребрах случайного гиперграфа $M G(S)$ ) $D_{M, n}=\frac{M}{n} \sum_{r=2}^{m} c_{r}(r)_{2}\left(n \sum_{s=1}^{n} p_{s}^{2}\right)$. Отсюда получаем оценку

$$
\mathbf{E} C \leq \sum_{l=3}^{n} \mathbf{E} C_{l} \leq \sum_{l=3}^{n} \frac{D_{M, n}^{l}}{2 l} .
$$

Из условия $M=o(n)$ и формулы (2) следует, что $D_{M, n}=O\left(\frac{M}{n} \sigma^{2} \sum_{r=2}^{m} c_{r}(r)_{2}\right)=o(1)$, поэтому $\mathbf{E} C=o(1)$. Утверждение теоремы 5 для $M G_{n, \bar{c}, \bar{p}}$ доказано. Следовательно, оно справедливо и для $M G(S)$.

Доказательство теоремы 6. В соответствии с теоремой 5 в условиях доказываемой теоремы почти все реализации случайного гиперграфа не 
имеют циклов. Оставшийся вид компонент - гипердеревья. Любое гипердерево из $l$ и более ребер содержит подграф, являющийся гипердеревом из $l$ ребер. Поэтому для доказательства теоремы достаточно показать отсутствие почти во всех реализациях случайного гиперграфа $M G_{n, \bar{c}, \bar{p}}$ гипердеревьев из $l$ ребер. Пусть $H-$ произвольное гипердерево из $l$ ребер. Пусть его ребра имеют ранги $r_{1}, \ldots, r_{l}$ и произведение вероятностей меток гиперграфа $H$ положительно: $\phi=\phi(H)>0$. Покажем, что в рассматриваемых условиях среднее значение случайной величины $\eta_{n}$, равной числу $H$-графов в случайном гиперграфе $M G_{n, \bar{c}, \bar{p}}$, удовлетворяет условию $\mathbf{E} \eta_{n}=o(1)$.

Через $I\left(\mathbf{i}_{1}, \ldots, \mathbf{i}_{l}, N_{1}, \ldots, N_{l}\right)$ будем обозначать индикатор события, заключающегося в том, что в случайном гиперграфе $M G_{n, \bar{c}, \bar{p}}$ имеется $H$-граф c $l$ ребрами, в котором $s$-е ребро имеет номер $N_{s}$ и состоит из вершин с индексами из упорядоченного множества $\mathbf{i}_{s}=\left\langle i_{s, 1}, \ldots, i_{s, r_{s}}\right\rangle, s=1, \ldots, l$. Для каждой пары наборов упорядоченных множеств $\left\langle\mathbf{i}_{1}, \ldots, \mathbf{i}_{l}\right\rangle$ и $\left\langle N_{1}, \ldots, N_{l}>\right.$, однозначно определяющей $H$-граф, справедлива оценка

$$
\mathbf{E} I\left(\mathbf{i}_{1}, \ldots, \mathbf{i}_{l}, N_{1}, \ldots, N_{l}\right)=\phi \prod_{s=1}^{l} \prod_{i \in \mathbf{i}_{s}} p_{i},
$$

где $\phi=c_{r_{1}} \ldots c_{r_{l}}-$ произведение вероятностей меток ребер гиперграфа $H$; $\prod_{s=1}^{l} \prod_{i \in i_{s}} p_{i}$ - вероятность того, что некоторые фиксированные $l$ ребер случайного гиперграфа $M G_{n, \bar{c}, \bar{p}}$ состоят из вершин, индексы которых образуют множества $\mathbf{i}_{1}, \ldots, \mathbf{i}_{l}$ соответственно. Число $H$-графов в случайном гиперграфе можно представить в виде

$$
\eta_{n}=\sum_{<\mathbf{i}_{1}, \ldots, \mathbf{i}_{l}><N_{1}, \ldots, N_{l}>} I\left(\mathbf{i}_{1}, \ldots, \mathbf{i}_{l}, N_{1}, \ldots, N_{l}\right) .
$$

В этой формуле суммирование осуществляется по упорядоченным наборам попарно не совпадающих номеров ребер $N_{1}, \ldots, N_{l}$ и множествам индексов $\mathbf{i}_{1}, \ldots, \mathbf{i}_{l}$, определяющим все возможные $H$-графы в реализациях случайного гиперграфа. Для гиперграфа $H$, имеющего порядок группы автоморфизмов $a(H)$, имеется ровно $a(H)$ различных пар наборов множеств индексов $\mathbf{i}_{1}, \ldots, \mathbf{i}_{l}$ и номеров ребер $N_{1}, \ldots, N_{l}$, определяющих один и тот же $H$-граф. Учитывая оценки $a(H) \geq 1, \phi \leq 1$, получаем формулу

$$
\mathbf{E} \eta_{n} \leq(M)_{l} \sum_{\left\langle\mathbf{i}_{1}, \ldots, \mathbf{i}_{l}>\right.} \prod_{s=1}^{l} \prod_{i \in \mathbf{i}_{s}} p_{i}
$$


Пусть гиперграф $H$ имеет $k$ вершин, из которых $k_{j}$ имеют степень $j$, $j=1, \ldots, l$. Так как $H$ гипердерево, то $k_{l}=0$ или $k_{l}=1$. Тогда справедлива формула

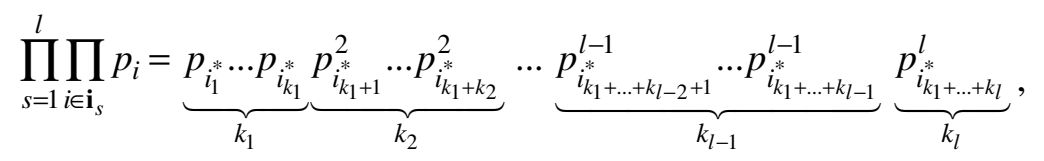

в правой части которой первая степень вероятностей появления вершин встречается $k_{1}$ раз, вторая степень $-k_{2}$ раз, ..., l-я степень $-k_{l}$ раз, a $i_{1}^{*}, \ldots, i_{k_{1}+\ldots+k_{l}}^{*}-$ соответствующим образом переупорядоченные индексы $i_{1,1}, \ldots, i_{1, r_{1}}, \ldots, i_{l, 1}, \ldots, i_{l, r_{l}}$, причем $k_{1}+\ldots+k_{l}=k$. Расширим диапазон суммирования в формуле (7). Пусть каждый индекс пробегает все $n$ возможных значений независимо от остальных индексов. Тогда получаем верхнюю оценку

$$
\mathbf{E} \eta_{n} \leq M^{l} \sum_{i_{1}^{*}} \sum_{i_{2}^{*}} \ldots \sum_{i_{k_{1}+\ldots+k_{l}}^{*}} p_{i_{1}^{*} \ldots} \ldots p_{i_{k_{1}}^{*}} p_{i_{k_{1}+1}^{*}}^{2} \ldots p_{i_{k_{1}+k_{2}}^{*}}^{2} \ldots p_{i_{k_{1}+\ldots+k_{l}+2}^{*}}^{l-1} \ldots p_{i_{k_{1}+\ldots+k_{l}-1}^{*}}^{l-1} p_{i_{k_{1}+\ldots+k_{l}}^{*}}^{l} .
$$

В этой формуле суммы по первым $k_{1}$ индексам $i_{1}^{*}, \ldots, i_{k_{1}}^{*}$ в соответствии с формулой (1) равны единице. Для остальных сумм по индексам $i_{k_{1}+1}^{*}, \ldots, i_{k_{1}+\ldots+k_{l}}^{*}$ справедливы оценки

$$
\sum_{k=1}^{n} p_{k}^{s} \leq\left(\frac{\varepsilon_{2}}{n}\right)^{s-2} \sum_{k=1}^{n} p_{k}^{2}, s=2, \ldots, l,
$$

откуда с учетом (2) имеем

$$
\sum_{k=1}^{n} p_{k}^{s}=O\left(n^{-(s-1)}\right), s=2, \ldots, l .
$$

Следовательно,

$$
\mathbf{E} \eta_{n}=O\left(M^{l} n^{-\sum_{s=2}^{l}(s-1) k_{s}}\right)
$$

Отсюда и из справедливых для гипердерева $H$ формул

$$
\sum_{s=1}^{l} l_{s}=l, \sum_{s=1}^{l} k_{s}=\sum_{i=1}^{m}(i-1) l_{i}+1=k, \sum_{s=1}^{l} s k_{s}=\sum_{i=1}^{m} i l_{i}
$$

следует соотношение

$$
\sum_{s=2}^{l}(s-1) k_{s}=\sum_{s=1}^{l} s k_{s}-k=\sum_{s=1}^{l} s k_{s}-\sum_{i=1}^{m}(i-1) l_{i}-1=\sum_{i=1}^{m} l_{i}-1=l-1,
$$


откуда $\mathbf{E} \eta_{n}=O\left(M^{l} n^{-(l-1)}\right)=o(1)$, так как $M=o\left(n^{1-1 / l}\right)$. Отсюда и из неравенства Маркова $\mathbf{P}\left(\eta_{n} \geq 1\right) \leq \mathbf{E} \eta_{n}$ для неотрицательной случайной величины $\eta_{n}$ и конечности числа различных (т. е. неизоморфных) гипердеревьев из $l$ ребер, имеющих ранги не более $m$, следует утверждение доказываемой теоремы для $M G_{n, \bar{c}, \bar{p}}$. В соответствии с установленной выше связью свойств $M G_{n, \bar{c}, \bar{p}}$ и $M G(S)$ отсюда следует справедливость утверждения и для $M G(S)$.

Доказательство теоремы 7. Доказательство проводится методом моментов, аналогично доказательствам теоремы 1.1 статьи [22] и теоремы 2a работы [33]. Основными отличиями условий доказываемой теоремы являются неоднородность случайного гиперграфа, упорядоченность вершин в его ребрах, наличие меток ребер, выбор вершин по схеме с возвращением, неравновероятный выбор неизвестных, случайный выбор метки из $E$ для каждого ребра. Кроме того, в указанных теоремах получены распределения чисел изолированных гипердеревьев и деревьев, а в формулировке данного утверждения такого ограничения нет. Однако в соответствии с теоремами 5 и 6 почти все реализации случайного гиперграфа в условиях доказываемой теоремы не имеют циклов и гипердеревьев $\mathrm{c} l+1$ и более ребрами. Это означает, что почти все реализации случайного гиперграфа имеют только изолированные гипердеревья с $l$ ребрами и предельные распределения чисел гипердеревьев $\mathrm{c} l$ ребрами и изолированных гипердеревьев $\mathrm{c} l$ ребрами совпадают. Поэтому для доказательства теоремы 7 достаточно получить аналогичный результат для случайных величин $\tau_{1}^{*}, \ldots, \tau_{t}^{*}$.

Доказательство довольно громоздко, поэтому сначала получим оценку среднего значения случайной величины $\tau_{k}^{*}=\tau_{k, n}^{*}$, равной числу изолированных $H$-графов-гипердеревьев в случайном гиперграфе $M G(S)$, где $H=H_{k}$.

Через $I^{*}\left(\mathbf{i}_{1}, \ldots, \mathbf{i}_{l}, N_{1}, \ldots, N_{l}\right)$ будем обозначать индикатор события, заключающегося в том, что в случайном гиперграфе $M G(S)$ имеется изолированный $H$-граф с $l$ ребрами, в котором $s$-е ребро имеет номер $N_{s}$ и состоит из вершин с индексами из упорядоченного множества $\mathbf{i}_{s}=\left\langle i_{s, 1}, \ldots, i_{s, r_{s}}\right\rangle$, $s=1, \ldots, l$. Для каждой пары $<\mathbf{i}_{1}, \ldots, \mathbf{i}_{l}>$ и $<N_{1}, \ldots, N_{l}>$ справедлива оценка

$$
\mathbf{E} I^{*}\left(\mathbf{i}_{1}, \ldots, \mathbf{i}_{l}, N_{1}, \ldots, N_{l}\right)=\phi \prod_{s=1}^{l} \prod_{i \in \mathbf{i}_{s}} p_{i} W\left(\mathbf{i}_{1}, \ldots, \mathbf{i}_{l}\right),
$$

где $\phi=c_{r_{1}, j_{1}, a_{1}} \ldots c_{r_{l}, j_{l}, a_{l}}$ - произведение вероятностей меток ребер гиперграфа $H$, равное вероятности того, что $l$ ребер случайного гиперграфа с выбранными номерами имеют метки $\left(r_{s}, j_{s}, a_{s}\right), s=1, \ldots, l$, совпадающие с метками гиперграфа $H$ (в частности, эти $l$ ребер имеют ранги $r_{1}, \ldots, r_{l}$ ); 
$\prod_{s=1}^{l} \prod_{i \in \mathbf{i}_{s}} p_{i}-$ вероятность того, что эти $l$ ребер состоят из вершин, индексы которых образуют множества $\mathbf{i}_{1}, \ldots, \mathbf{i}_{l}$; величина $W\left(\mathbf{i}_{1}, \ldots, \mathbf{i}_{l}\right)$ равна вероятности того, что $H$-граф на множестве вершин $\mathbf{i}_{1}, \ldots, \mathbf{i}_{l}$ является изолированным в случайном гиперграфе, и определяется формулой

$$
W\left(\mathbf{i}_{1}, \ldots, \mathbf{i}_{l}\right)=\sum_{M_{1}^{*}+\ldots+M_{m}^{*}=M-l} \frac{(M-l) !}{M_{1}^{*} ! \ldots, M_{m}^{*} !} c_{1}^{M_{1}^{*}} \ldots c_{m}^{M_{m}^{*}}\left(\sum_{j \notin\left\{\mathbf{i}_{1} \cup \ldots \cup \mathbf{i}_{l}\right\}} p_{j}\right)^{\sum_{i=1}^{m} i M_{i}^{*}} .
$$

В этой формуле осуществляется перебор $M-l$ ребер гиперграфа, оставшихся после выбора $l$ ребер для $H$-графа; суммирование по $M_{1}^{*}, \ldots, M_{m}^{*}$ соответствует перебору всех возможных вариантов ребер ранга $1, \ldots, m$; величина $\frac{(M-l) !}{M_{1}^{*} ! \ldots, M_{m}^{*} !} c_{1}^{M_{1}^{*}} \ldots c_{m}^{M_{m}^{*}}$ равна вероятности наличия в рассматриваемых $M-l$ ребрах случайного гиперграфа $M_{s}^{*}$ ребер ранга $s, s=1, \ldots, m$; последний множитель в формуле для $W\left(\mathbf{i}_{1}, \ldots, \mathbf{i}_{l}\right)$ равен вероятности того, что $M-l$ ребер случайного гиперграфа, не принадлежащих $H$-графу, не инцидентны множеству вершин $\left\{\mathbf{i}_{1} \cup \ldots \bigcup \mathbf{i}_{l}\right\}$.

Оценим величину $W\left(\mathbf{i}_{1}, \ldots, \mathbf{i}_{l}\right)$. С помощью формулы (1) и соотношения $\left|\mathbf{i}_{1}\right|+\ldots+\left|\mathbf{i}_{l}\right|=r_{1}+\ldots+r_{l}$ убеждаемся в справедливости оценки

$$
\left(1-\frac{\varepsilon_{2}\left(r_{1}+\ldots+r_{l}\right)}{n}\right)^{M-l} \leq\left(\sum_{j \neq\left\{\mathbf{i}_{1} \cup \ldots \cup \mathrm{i}_{l}\right\}} p_{j}\right)^{\sum_{i=1}^{m} i M_{i}^{*}} \leq\left(1-\frac{\varepsilon_{1}\left(r_{1}+\ldots+r_{l}\right)}{n}\right)^{M-l} \leq 1 .
$$

Верхняя и нижняя оценки в этой формуле не зависят от наборов $\mathbf{i}_{1}, \ldots, \mathbf{i}_{l}$, и при условии $M \sim c n^{1-1 / l}$ теоремы ввиду конечности параметра $l$ и суммы $r_{1}+\ldots+r_{l}$ выполняется соотношение $\left(1-\frac{\varepsilon_{1}\left(r_{1}+\ldots+r_{l}\right)}{n}\right)^{M-l}=1+O\left(n^{-1 / l}\right)$. Отсюда и из формулы

$$
\sum_{\sum_{j=1}^{m} M_{s}^{*}=M-l} \frac{(M-l) !}{M_{1}^{*} ! \ldots, M_{m}^{*} !} c_{1}^{M_{1}^{*}} \ldots c_{m}^{M_{m}^{*}}=1
$$

получаем оценку $W\left(\mathbf{i}_{1}, \ldots, \mathbf{i}_{l}\right)=1+o(1)$, равномерную по $r_{1}, \ldots, r_{l}$.

Для числа $H$-графов, являющихся изолированными гипердеревьями, справедлива формула

$$
\tau^{*}=\sum_{\left\langle\mathbf{i}_{1}, \ldots, \mathbf{i}_{l}><N_{1}, \ldots, N_{l}\right\rangle} I^{*}\left(\mathbf{i}_{1}, \ldots, \mathbf{i}_{l}, N_{1}, \ldots, N_{l}\right),
$$


где суммирование осуществляется по упорядоченным наборам попарно не совпадающих номеров ребер $N_{1}, \ldots, N_{l}$ и множествам индексов $\mathbf{i}_{1}, \ldots, \mathbf{i}_{l}$, определяющим все возможные $H$-графы в реализациях случайного гиперграфа.

Покажем, что порядок группы автоморфизмов гипердерева $H\left(H=H_{k}\right.$, $k=1, \ldots, t)$ равен единице. Действительно, при $l>2$ ребро с номером $l$ при автоморфизме гипердерева может перейти только в себя, так как из всех ребер гипердерева $H$ только оно инцидентно $l-1$ ребрам (см. рис. 1); ввиду упорядоченности вершин в ребрах каждая вершина $l$-го ребра может перейти только в себя, в том числе вершина, инцидентная $s$-му ребру гипердерева, $s=1, \ldots, l-1$; из последнего следует, что каждое ребро гипердерева с номером $s, s=1, \ldots, l-1$ тоже может перейти только в себя. Если при $l=2$ метки $\left(r_{1}, j_{1}, a_{1}\right),\left(r_{2}, j_{2}, a_{2}\right)$ ребер гипердерева $H$ различны, то порядок его группы автоморфизмов тоже равен единице, так как при изоморфизме метки ребер сохраняются. Если же эти метки одинаковы, то общей вершине двух ребер гипердерева $H$ присвоены разные номера в его двух ребрах, так как оно соответствует несовместной подсистеме уравнений. Отсюда следует, что порядок группы автоморфизмов гипердерева $H$ при $l=2$ и в этом случае равен единице.

С учетом оценки для $W\left(\mathbf{i}_{1}, \ldots, \mathbf{i}_{l}\right)$ и формулы $a(H)=1$ получаем соотношение

$$
\mathbf{E} \tau^{*}=(M)_{l} \phi \sum_{<\mathbf{i}_{1}, \ldots, \mathbf{i}_{l}>} \prod_{s=1}^{l} \prod_{i \in \mathbf{i}_{s}} p_{i}(1+o(1)) .
$$

Обозначим через $s_{l, 1} \ldots, s_{l, l-1}$ номера вершин в $l$-м ребре $\mathbf{i}_{l}=<i_{l, 1}, \ldots, i_{l, r_{l}}>$ $H$-графа, инцидентных первому, второму, ..., $(l-1)$-му ребрам этого $H$-графа. В слагаемых рассматриваемой суммы по $\mathbf{i}_{1}, \ldots, \mathbf{i}_{l}$ вероятности с индексами $s_{l, 1} \ldots, s_{l, l-1}$ встречаются дважды, с остальными индексами - по одному разу. Сумму по $\mathbf{i}_{1}, \ldots, \mathbf{i}_{l}$ заменим на $r_{1}+\ldots+r_{l}-l+1$ вложенных сумм по индексам $i_{1,1}, \ldots, i_{l, r_{l}}$. Аналитический вид получаемого выражения очень громоздок и не обязателен для получения асимптотической оценки рассматриваемой суммы, поэтому ограничимся описанием этого выражения. В суммах по индексам $s_{l, j}, j=1, \ldots, l-1$, слагаемые равны $p_{s_{l, j}}^{2}$, а в суммах по остальным индексам слагаемые являются аналогичными вероятностями, но в первой степени. Перебор индексов $i_{1,1}, \ldots, i_{l, r_{l}}$ осуществляется с учетом их порядка. Первый индекс $i_{1,1}$ пробегает все возможные значения $1, \ldots, n$. Выбор значений второго индекса зависит от значения первого индекса: перебираются все возможные $n$ значений, за исключением значения первого индекса. Далее перебор индексов для вершин аналогичен: перебираются все возможные $n$ значений, за исключением выбранных до этого значений пре- 
дыдущих индексов. Единственное отличие появляется только для индексов $l$-го ребра $s_{l, 1}, \ldots, s_{l, l-1}$, которые встречаются в исходной сумме дважды. Они уже включены в суммы при переборе индексов для первых $l-1$ ребер (слагаемые сумм имеют вид квадратов вероятностей), поэтому при переборе индексов для $l$-го ребра они пропускаются.

С учетом формулы (1) каждую сумму с первыми степенями вероятностей можно оценить сверху величиной $\sum_{j=1}^{n} p_{j}=1$, снизу - величиной $\sum_{j=1}^{n} p_{j}-\frac{\varepsilon_{2}\left(r_{1}+\ldots+r_{l}-l\right)}{n}=1+\alpha_{1}(n), \quad$ в которой $\quad \alpha_{1}(n)=O\left(n^{-1}\right)=o(1), \quad r_{1}+\ldots+$ $+r_{l}-l$ - максимально возможное число индексов, которые были в предыдущих суммах (может достигаться при переборе последнего индекса $l$-го ребpa). Суммы по индексам $s_{l, j}, j=1, \ldots, l-1$ оцениваются аналогично, с учетом формул (1), (2): сверху величиной $\sum_{j=1}^{n} p_{j}^{2}$, снизу — величиной

$$
\sum_{j=1}^{n} p_{j}^{2}-\left(r_{1}+\ldots+r_{l}-l\right)\left(\frac{\varepsilon_{2}}{n}\right)^{2}=\sum_{j=1}^{n} p_{j}^{2}+\alpha_{2}(n)=n^{-1}\left(\sigma^{2}+O\left(n^{-1}\right)\right),
$$

где $\alpha_{2}(n)=O\left(n^{-2}\right)$. Собрав приведенные оценки, получаем

$$
(M)_{l} \phi\left(\sum_{j=1}^{n} p_{j}^{2}+\alpha_{2}(n)\right)^{l-1}\left(1+\alpha_{1}(n)\right)^{r_{1}+\ldots+r_{l}} \leq \mathbf{E} \tau^{*} \leq(M)_{l} \phi\left(\sum_{j=1}^{n} p_{j}^{2}\right)^{l-1} .
$$

Так как при $M \sim c n^{1-1 / l}$

$$
(M)_{l}\left(\sum_{j=1}^{n} p_{j}^{2}+\alpha_{2}(n)\right)^{l-1} \sim \frac{M^{l}}{n^{l-1}}\left(\sigma^{2}+O\left(n^{-1}\right)\right)^{l-1} \sim c^{l} \sigma^{2(l-1)},
$$

то справедливы асимптотические оценки

$$
\mathbf{E} \tau^{*}=\mathbf{E} \tau_{k}^{*} \sim c^{l} \phi \sigma^{2(l-1)}=\lambda=\lambda_{k}, k=1, \ldots, t .
$$

Теперь докажем сходимость всех смешанных факториальных моментов

$$
\mathbf{E}\left(\left(\tau_{1}^{*}\right)_{u_{1}} \ldots\left(\tau_{t}^{*}\right)_{u_{t}}\right)=\mathbf{E}\left(\tau_{1}^{*}\left(\tau_{1}^{*}-1\right) \ldots\left(\tau_{1}^{*}-u_{1}+1\right) \ldots \tau_{t}^{*}\left(\tau_{t}^{*}-1\right) \ldots\left(\tau_{t}^{*}-u_{t}+1\right)\right)
$$

порядка $u=2,3, \ldots, u_{1}+\ldots+u_{t}=u$, векторной случайной величины $\left(\tau_{1}^{*}, \ldots, \tau_{t}^{*}\right)$ к соответствующим смешанным факториальным моментам распределения $P\left(\lambda_{1}, \ldots, \lambda_{t}\right)$. Рассмотрим $t$ групп подграфов в реализациях случайного гиперграфа $M G(S)$. Пусть $k$-я группа состоит из $u_{k} H_{k}$-графов, $k=1, \ldots, t$. В этой группе $H_{k}$-граф с номером $v=v(k)$ однозначно определяется набором упоря- 
доченных множеств $\mathbf{i}^{(k, v)}=\left\{\mathbf{i}_{1}^{(k, v)}, \ldots, \mathbf{i}_{l}^{(k, v)}\right\}$, где $\mathbf{i}_{1}^{(k, v)}=<i_{1,1}^{(k, v)}, \ldots, i_{1, r_{1}^{(k)}}^{(k, v)}>, \ldots, \mathbf{i}_{l}^{(k, v)}=$ $=<i_{l, 1}^{(k, v)}, \ldots, i_{l, r_{l}^{(k)}}^{(k, v)}>$ и набором номеров его ребер $N_{1}^{(k, v)}, \ldots, N_{l}^{(k, v)}, v=1, \ldots, u_{k}$.

Обозначения множеств и их элементов аналогичны введенным выше $\mathbf{i}_{s}, i_{s, j}$. Первый добавленный верхний индекс $k$ в $\mathbf{i}_{s}^{(k, v)}, i_{s, j}^{(k, v)}$ и $N_{s}^{(k, v)}$ соответствует номеру $k$ гиперграфа $H_{k}, k=1, \ldots, t$, второй верхний индекс $v$ соответствует номеру гиперграфа в группе из $u_{k} H_{k}$-графов. Обозначим через

$$
I^{*}\left(\mathbf{i}^{(1,1)}, \ldots, \mathbf{i}^{\left(1, u_{1}\right)}, \ldots, \mathbf{i}^{(t, 1)}, \ldots, \mathbf{i}^{\left(t, u_{t}\right)}, N_{1}^{(1,1)}, \ldots, N_{l}^{(1,1)}, \ldots, N_{1}^{\left(t, u_{t}\right)}, \ldots, N_{l}^{\left(t, u_{t}\right)}\right)
$$

случайную величину, равную индикатору события, заключающегося в том, что в $M G(S)$ имеется $u_{k}$ изолированных $H_{k}$-графов с ребрами, имеющими индексы в множествах $\left\{\mathbf{i}_{1}^{(k, 1)}, \ldots, \mathbf{i}_{l}^{(k, 1)}\right\}, \ldots,\left\{\mathbf{i}_{1}^{\left(k, u_{k}\right)}, \ldots, \mathbf{i}_{l}^{\left(k, u_{k}\right)}\right\}$, и номера ребер $N_{1}^{(k, v)}, \ldots, N_{l}^{(k, v)}$, $k=1, \ldots, t$. Тогда (см., например, [12], [18])

$$
\mathbf{E}\left(\left(\tau_{1}^{*}\right)_{u_{1}} \ldots\left(\tau_{t}^{*}\right)_{u_{t}}\right)=\sum I^{*}\left(\mathbf{i}^{(1,1)}, \ldots, \mathbf{i}^{\left(t, u_{t}\right)}, N_{1}^{(1,1)}, \ldots, N_{l}^{\left(t, u_{t}\right)}\right),
$$

где суммирование проводится по всем упорядоченным наборам различных индексов $\mathbf{i}^{(1,1)}, \ldots, \mathbf{i}^{\left(t, u_{t}\right)}$ и различных номеров ребер $N_{1}^{(1,1)}, \ldots, N_{l}^{\left(t, u_{t}\right)}$, определяющих $u_{k} H_{k}$-графов, $k=1, \ldots, t$. Как и при оценке $\mathbf{E} \tau^{*}=\mathbf{E} \tau_{k}^{*}$, убеждаемся в справедливости формулы

$$
\begin{aligned}
& \mathbf{E}\left(\left(\tau_{1}^{*}\right)_{u_{1}} \ldots\left(\tau_{t}^{*}\right)_{u_{t}}\right)=\frac{\left(\varphi^{(1)}\right)^{u_{1}} \ldots\left(\varphi^{(t)}\right)^{u_{t}}}{\left(a\left(H_{1}\right)\right)^{u_{1}} \ldots\left(a\left(H_{t}\right)\right)^{u_{t}}} \times \\
& \times \sum_{\left\langle\mathbf{i}_{1}^{(1,1)},, \ldots \mathbf{i}_{l}^{\left(t, u_{t}\right)}\right\rangle} \sum_{\left\langle N_{1}^{(1,1)}, \ldots, N_{l}^{\left(t, u_{t}\right)}\right\rangle}\left(\prod_{k=1}^{t} \prod_{v=1}^{u_{k}} \prod_{s=1}^{l} \prod_{i \in \mathbf{i}_{s}^{(k, v)}} p_{i} W\left(\mathbf{i}_{1}^{(1,1)}, \ldots, \mathbf{i}_{l}^{\left(t, u_{t}\right)}\right)\right),
\end{aligned}
$$

в которой суммирование осуществляется по таким множествам $\mathbf{i}_{1}^{(1,1)}, \ldots, \mathbf{i}_{l}^{\left(t, u_{t}\right)}$, что первая, вторая, ..., $u_{1}$-я группы по $l$ множеств из первых $l u_{1}$ множеств индексов на соответствующих множествах вершин образуют $H_{1}$-графы с номерами ребер $N_{1}^{(1,1)}, \ldots, N_{l}^{\left(1, u_{1}\right)}, \ldots$, первая, вторая, $\ldots, u_{t}$-я группы по $l$ множеств из последних $l u_{t}$ множеств индексов на соответствующих множествах вершин образуют $H_{t}$-графы с номерами ребер $N_{1}^{(t, 1)}, \ldots, N_{l}^{\left(t, u_{t}\right)}$, причем любые два из этих подграфов не имеют общих вершин. Величина $\prod_{k=1}^{t} \prod_{v=1}^{u_{k}} \prod_{s=1}^{l} \prod_{i \in \mathbf{i}_{s}^{(k, v)}} p_{i}$ равна вероятности того, что в случайном гиперграфе $M G(S)$ есть ребра с множествами индексов $\mathbf{i}_{1}^{(1,1)}, \ldots, \mathbf{i}_{l}^{\left(t, u_{t}\right)}$, образующих $l u_{k}$ рассматриваемых $H_{k}$-графов, $k=1, \ldots, t$. Здесь произведение по индексу $k$ соответству- 
ет перебору $t$ гипердеревьев $H_{1}, \ldots, H_{t}$, произведение по индексу $v$ для каждого $k$ перебирает $u_{k} H_{k}$-графов, произведение по индексу $s$ перебирает $l$ ребер каждого из этих $H_{k}$-графов, внутреннее произведение по индексу $i$ перебирает вершины в каждом из ребер $H_{k}$-графа. Величина $\phi^{(k)}=\prod_{s=1}^{l} c_{r_{s}^{(k)}, j_{s}^{(k)}, a_{s}^{(k)}}-$ произведение вероятностей меток ребер гиперграфа $H_{k}$, а $a\left(H_{k}\right)$ - порядок его группы автоморфизмов, $k=1, \ldots, t$. Число $(M)_{l\left(u_{1}+\ldots+u_{t}\right)}$ равно количеству вариантов выбора $l\left(u_{1}+\ldots+u_{t}\right)$ различных номеров ребер $N_{1}^{(1,1)}, \ldots, N_{l}^{\left(t, u_{t}\right)}$ из $M$ возможных для $u_{k}$ рассматриваемых $H_{k}$-графов, $k=1, \ldots, t$; множитель $\left(\varphi^{(1)}\right)^{u_{1}} \ldots\left(\varphi^{(t)}\right)^{u_{t}}$ равен вероятности того, что эти $l\left(u_{1}+\ldots+u_{t}\right)$ ребер случайного гиперграфа с выбранными номерами имеют метки $\left(r_{s}^{(k)}, j_{s}^{(k)}, a_{s}^{(k)}\right)$, $k=1, \ldots, t$, совпадающие с метками $u_{k}$ гиперграфов $H_{k}, k=1, \ldots, t$. Величина $W\left(\mathbf{i}_{1}^{(1,1)}, \ldots, \mathbf{i}_{l}^{\left(t, u_{t}\right)}\right)$ есть вероятность изолированности каждого из рассматриваемых $u_{1}+\ldots+u_{t}$ подграфов в $M G(S)$, равная

$$
\sum_{j=1}^{m} M_{s=M-l\left(u_{1}+\ldots+u_{t}\right)} \frac{\left(M-l\left(u_{1}+\ldots+u_{t}\right)\right) !}{M_{1}^{*} ! \ldots, M_{m}^{*} !} c_{1}^{M_{1}^{*} \ldots} c_{m}^{M_{m}^{*}}\left(\sum_{j \notin\left\{\mathbf{i}_{1}^{(1,1)}, \ldots, \mathbf{i}_{l}^{\left(,, t_{t}\right)}\right\}} p_{j}\right)^{\sum_{i=1}^{m} i M_{i}^{*}} .
$$

Последняя формула аналогична формуле для $W\left(\mathbf{i}_{1}, \ldots, \mathbf{i}_{l}\right)$, полученной выше. Отличие заключается в переборе $M-l\left(u_{1}+\ldots+u_{t}\right)$, а не $M-l$ ребер. Проводя необходимые преобразования, как и при оценке $\mathbf{E} \tau_{k}^{*}$, убеждаемся в том, что для любого натурального $t$ и любых целых неотрицательных чисел $u_{1}, \ldots, u_{t}$

$$
\mathbf{E}\left(\left(\tau_{1}^{*}\right)_{u_{1}} \ldots\left(\tau_{t}^{*}\right)_{u_{t}}\right) \rightarrow \lambda_{1}^{u_{1}} \ldots \lambda_{t}^{u_{t}} .
$$

Следовательно, все смешанные факториальные моменты векторной случайной величины $\left(\tau_{1}^{*}, \ldots, \tau_{t}^{*}\right)$ сходятся к соответствующим моментам многомерного распределения Пуассона с независимыми компонентами и приведенными в условии доказываемой теоремы средними значениями. Так как многомерное распределение Пуассона однозначно определяется своими моментами, то распределение векторной случайной величины $\left(\tau_{1}^{*}, \ldots, \tau_{t}^{*}\right)$ сходится к многомерному распределению Пуассона с вектором средних значений $\left(\lambda_{1}, \ldots, \lambda_{t}\right)$ (см. теорему 21 главы 1 книги [30]). Доказательство утверждения теоремы о сходимости распределения векторной случайной величины $\left(\tau_{1}, \ldots, \tau_{t}\right)$ завершает сделанное в начале доказательства 
замечание о совпадении предельных распределений чисел гипердеревьев и чисел изолированных гипердеревьев в случайном гиперграфе $M G(S)$. Вторая часть утверждения о сходимости распределения суммы компонент этой векторной случайной величины следует из аддитивного свойства распределения Пуассона.

Пусть $Q$ и $Q^{*}$ - некоторые свойства случайной системы уравнений (случайного гиперграфа $M G(S)), \bar{Q}-$ свойство, дополнительное свойству $Q$ (им обладают реализации, не обладающие свойством $Q)$, а $\mathbf{P}\left(Q^{*} \mid Q\right)$ - условная вероятность наличия свойства $Q^{*}$ при условии, что имеет место свойство $Q$.

Лемма 2. Если $\lim _{n \rightarrow \infty} \mathbf{P}(Q)=1$, то $\lim _{n \rightarrow \infty} \mathbf{P}\left(Q^{*}\right)$ существует тогда и только тогда, когда существует $\lim _{n \rightarrow \infty} \mathbf{P}\left(Q^{*} \mid Q\right)$. Если последние два предела существуют, то они совпадают.

Доказательство леммы 2. Так как $\mathbf{P}(\bar{Q})=o(1)$, то $\mathbf{P}\left(Q^{*} \cap \bar{Q}\right)=o(1)$. Утверждение следует из формулы

$$
\mathbf{P}\left(Q^{*} \mid Q\right)=\frac{\mathbf{P}\left(Q^{*} \cap Q\right)}{\mathbf{P}(Q)}=\frac{\mathbf{P}\left(Q^{*}\right)-\mathbf{P}\left(Q^{*} \cap \bar{Q}\right)}{\mathbf{P}(Q)}=\frac{\mathbf{P}\left(Q^{*}\right)+o(1)}{1+o(1)}=\mathbf{P}\left(Q^{*}\right)+o(1) .
$$

Доказательство следствия 3. Воспользуемся леммой 2 для случайного гиперграфа $M G(S)$. Пусть свойство $Q$ заключается в отсутствии особых ребер в реализациях $M G(S)$. В соответствии с теоремой 5 при $M=o(n)$ почти все реализации $M G(S)$ не имеют циклов, в том числе - особых ребер. Поэтому условие леммы 2 выполнено. Для распространения утверждений теорем 5, 6 на случайный гиперграф с бесповторной выборкой вершин в ребрах достаточно выбрать свойства $Q^{*}$, заключающиеся соответственно в отсутствии циклов и гипердеревьев $\mathrm{c} l$ и более ребрами в реализациях случайного гиперграфа $M G(S), l \geq 2$. В соответствии с леммой 2 для этих свойств выполняется соотношение $\lim _{n \rightarrow \infty} \mathbf{P}\left(Q^{*} \mid Q\right)=\lim _{n \rightarrow \infty} \mathbf{P}\left(Q^{*}\right)$ при $M=o(n)$ и при $M=o\left(n^{1-1 / l}\right)$ соответственно, $l \geq 2$.

Для распространения утверждения теоремы 7 на случайный гиперграф с бесповторной выборкой вершин в ребрах выберем свойства $Q_{j_{1}, \ldots, j_{t}}^{*}$, заключающиеся в наличии в $M G(S)$ ровно $j_{k}$ рассматриваемых в теореме 7 $H_{k}$-графов-гипердеревьев с $l$ ребрами, $j_{k}=0,1, \ldots, k=1, \ldots, t$. В соответствии с теоремой 7 и леммой 2 при $M \sim c n^{1-1 / l}$ справедлива оценка

$$
\lim _{n \rightarrow \infty} \mathbf{P}\left(Q_{j_{1}, \ldots, j_{t}}^{*} \mid Q\right)=\lim _{n \rightarrow \infty} \mathbf{P}\left(Q_{j_{1}, \ldots, j_{t}}^{*}\right)=\prod_{k=1}^{t} e^{-\left(\lambda_{1}+\ldots+\lambda_{t}\right)} \frac{\left(\lambda_{1}+\ldots+\lambda_{t}\right)^{j_{k}}}{j_{k} !} .
$$


Отсюда следует первая часть утверждения теоремы 7. Вторая часть о распределении общего числа гипердеревьев доказывается аналогично с использованием свойств $Q_{j}^{*}, j=0,1, \ldots$, заключающихся в наличии

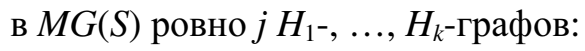

$$
\lim _{n \rightarrow \infty} \mathbf{P}\left(Q_{j}^{*} \mid Q\right)=\lim _{n \rightarrow \infty} \mathbf{P}\left(Q_{j}^{*}\right)=e^{-\left(\lambda_{1}+\ldots+\lambda_{t}\right)} \frac{\left(\lambda_{1}+\ldots+\lambda_{t}\right)^{j}}{j !}, j=0,1, \ldots
$$

\section{3. Доказательства основных результатов}

Из теорем 5-7 следует, что случайный гиперграф $M G(S)$, соответствующий случайной системе уравнений $S$, имеет следующие свойства. При $M=o(n)$ почти все его реализации не имеют циклов (в частности, особых ребер), т. е. состоят только из гипердеревьев. При $M=o\left(n^{1-1 / l}\right)$ почти все реализации $M G(S)$ не имеют также гипердеревьев, состоящих из $l$ или более ребер. При $M \sim c n^{1-1 / l}$ в $M G(S)$ числа гипердеревьев с $l$ ребрами, которые рассматриваются в теореме 7 , имеют предельным распределение $P\left(\lambda_{1}, \ldots, \lambda_{t}\right)$, где $\lambda_{k}=c^{l} \phi^{(k)} \sigma^{2(l-1)}, k=1, \ldots, t$, а предел вероятности отсутствия таких гипердеревьев в $M G(S)$ равен $\exp \left\{-c^{l} \sigma^{2(l-1)} \sum_{k=1}^{t} \phi^{(k)}\right\}$. При неограниченном росте параметра $c$ эта вероятность стремится к нулю, поэтому при $M / n^{1-1 / l} \rightarrow \infty$ почти все реализации $M G(S)$ имеют гипердеревья с $l$ ребрами рассмотренного в теореме 7 вида. В реализациях $M G(S)$ могут появляться, вообще говоря, и другие гипердеревья с $l$ или менее ребрами.

Доказательство теоремы 1. Пусть выполнены условия $c^{(b)}>0, R^{(b)}=$ $=r-1$ доказываемой теоремы. Тогда из уравнений, допустимых в случайной системе уравнений $S$, можно построить одну или более несовместных подсистем уравнений, описанных в лемме 1 . В теореме 7 изучено предельное распределение чисел подграфов в $M G(S)$, соответствующих именно таким подсистемам в реализациях $S$. Следовательно, при $M / n^{1-1 / r} \rightarrow \infty$ почти все реализации $M G(S)$ имеют гипердеревья с $r$ ребрами приведенного вида, и почти все реализации $S$ несовместны, так как имеют несовместные подсистемы, соответствующие таким гипердеревьям. Если же $M=o\left(n^{1-1 / n}\right)$, то почти все реализации $M G(S)$ не имеют гипердеревьев, состоящих из $r$ или более ребер. Однако в соответствии с леммой 1 такие гипердеревья соответствуют только совместным подсистемам случайной системы уравнений $S$. Следовательно, при $M=o\left(n^{1-1 / r}\right)$ почти все реализации $S$ совместны. Таким образом, доказано, что условия $c^{(b)}>0, R^{(b)}=r-1$ достаточны для наличия пороговой функции совместности вида $n^{1-1 / r}=n^{1-1 /\left(R^{(b)}+1\right)}, \quad 2 \leq r \leq m+1$, у случайной системы уравнений $S$. 
Перейдем к доказательству необходимости условий $c^{(b)}>0, R^{(b)}=r-1$, $b \in B$, для наличия пороговой функции совместности вида $n^{1-1 / r}$, $2 \leq r \leq m+1$, у случайной системы уравнений $S$. Пусть $S$ имеет такую пороговую функцию совместности. Предположим, что $c^{(b)}=0$ для каждого $b \in B$. Тогда уравнения реализаций $S$ не имеют однозначно определяющихся неизвестных. В теореме 5 показано, что при $M=o(n)$ почти все реализации $M G(S)$ состоят только из гипердеревьев. Однако в соответствии с утверждением леммы 2 статьи [25] любая подсистема уравнений совместна, если она состоит из уравнений без однозначно определяющихся неизвестных и соответствует гипердереву. Тогда почти все реализации случайной системы уравнений $S$ совместны при $M=o(n)$ и она не может иметь пороговую функцию совместности вида $n^{1-1 / r}$. Следовательно, предположение $c^{(b)}=0$ для каждого $b \in B$ неверно и справедливо условие $c^{(b)}>0$ для некоторого $b \in B$.

Покажем необходимость условия $R^{(b)}=r-1,2 \leq r \leq m+1$. Из определения величины $R^{(b)}$ и того, что каждое уравнение реализаций $S$ содержит от $m$ или менее неизвестных, следует, что $R^{(b)}$ может принимать только значения $0,1, \ldots, m$. В случае $R^{(b)}=0$ в соответствии со следствием 1 все реализации случайной системы уравнений $S$ совместны и имеют решение $\mathbf{b}$. Это противоречит тому, что $S$ имеет пороговую функцию совместности. Следовательно, справедлива оценка $1 \leq R^{(b)} \leq m$. Таким образом, для системы $S$ с пороговой функцией совместности $n^{1-1 / r}$ доказана необходимость выполнения условий $c^{(b)}=0$ и $1 \leq R^{(b)} \leq m$ для некоторого $b \in B$. Выше показано, что эти условия достаточны для того, чтобы $S$ имела пороговую функцию совместности $n^{1-1 /\left(R^{(b)}+1\right)}$. Следовательно, $n^{1-1 / r}=n^{1-1 /\left(R^{(b)}+1\right)}$ и $R^{(b)}=r-1$. Необходимость условий $c^{(b)}>0, R^{(b)}=r-1, b \in B$ для наличия пороговой функции совместности $n^{1-1 / r}, 2 \leq r \leq m+1$, у случайной системы уравнений $S$ доказана.

Перейдем коценке вероятности совместности случайной системы уравнений $S$ при условии (4). В лемме 1 приведен вид несовместных подсистем, которые с ненулевой предельной вероятностью могут появляться в реализация $S$. В левой части $k$-го уравнения таких подсистем стоит функция $f_{i_{k}, j_{k}}$, в правой - элемент $a_{k}, k=1, \ldots, r$, причем $c_{i_{k}, j_{k}, a_{k}} t_{i_{k}, j_{k}, a_{k}}^{(b)}>0$ для первых $r-1$ уравнений и $c_{i_{r}, j_{r}, a_{r}} k_{i_{r}, j_{r}, a_{r}}^{(b, r-1)}>0$ для последнего уравнения. Первые $r-1$ уравнений попарно не имеют общих неизвестных, но каждое их них имеет ровно одно общее неизвестное с $r$-м уравнением. В каждом из этих $r$ - 1 уравнений общие с $r$-м уравнением неизвестные однозначно определяются и равны $b$, а номера совпадающих с ними неизвестных $r$-го уравнения образуют несовместную $t$-фиксацию значением $b$ уравнения (3) при 
$f_{i, j}=f_{i_{k}, j_{k}}$ и $a=a_{k}$. Из условий $c^{(b, r-1)}>0$ (так как $\left.R^{(b)}=r-1\right)$ и $c^{(b)}>0$ следует, что множество подсистем такого вида не пусто. Несовместность таких подсистем следует из их определения. Из условия $c_{i_{r}, j_{r}, a_{r}} \prod_{k=1}^{r-1} c_{i_{k}, j_{k}, a_{k}}>0$ следует, что они допустимы в реализациях рассматриваемой случайной системы уравнений.

Каждой из рассматриваемых подсистем уравнений соответствует гипердерево с порядком группы автоморфизмов, равным 1, так как вершины во всех ребрах упорядочены. Поэтому число таких гипердеревьев с учетом фиксированного порядка первых ребер $-k_{i_{r}, j_{r}, a_{r}}^{(b, r-1)} \prod_{k=1}^{r-1} t_{i_{k}, j_{k}, a_{k}}^{(b)}$, где $k_{i_{r}, j_{r}, a_{r}}^{(b, r-1)}-$ число несовместных $(r-1)$-фиксаций значением $b$ неизвестных уравнения (3) при $(i, j, a)=\left(i_{r}, j_{r}, a_{r}\right), t_{i_{k}, j_{k}, a_{k}}^{(b)}-$ число неизвестных в уравнении (3) при $(i, j, a)=\left(i_{k}, j_{k}, a_{k}\right), k=1, \ldots, r-1$, которые однозначно определяются из него и равны $b$. В соответствии с теоремой 7 распределение числа всех таких гипердеревьев в случайном гиперграфе $M G(S)$ сходится к распределению Пуассона со средним значением, определяемым следующей формулой, в которой ведется суммирование по всем упорядоченным наборам $\left(i_{k}, j_{k}, a_{k}\right), k=1, \ldots, r:$

$$
\begin{aligned}
& c^{r} \sigma^{2(r-1)} \sum_{\left(i_{r}, j_{r}, a_{r}\right)} c_{i_{r}, j_{r}, a_{r}} k_{i_{r}, j_{r}, a_{r}}^{(b, r-1)} \sum_{\substack{\left(i_{1}, j_{1}, a_{1}\right), \ldots \\
\left(i_{r-1}, j_{r-1}, a_{r-1}\right)}}\left(c_{i_{1}, j_{1}, a_{1}} t_{i_{1}, j_{1}, a_{1}}^{(b)} \ldots c_{i_{r-1}, j_{r-1}, a_{r-1}} t_{i_{r-1}, j_{r-1}, a_{r-1}}^{(b)}\right)= \\
& =c^{r} \sum_{\left(i_{r}, j_{r}, a_{r}\right)} c_{i_{r}, j_{r}, a_{r}} k_{i_{r}, j_{r}, a_{r}}^{(b, r-1)}\left(\sigma^{2} \sum_{(i, j, a) \in E} c_{i, j, a} t_{i, j, a}^{(b)}\right)^{r-1}=c^{r} c^{(b, r-1)}\left(\sigma^{2} c^{(b)}\right)^{r-1}>0 .
\end{aligned}
$$

Отсюда следуют приведенные в утверждении теоремы 1 оценки для $\lambda$ и для вероятности совместности $\mathbf{P}_{c}(S)$ случайной системы уравнений.

Доказательство теоремы 4. Из теорем 5, 6 следует, что при условии (4) почти все реализации случайной системы уравнений $S$ состоят из подсистем уравнений, соответствующих гипердеревьям из $r$ или менее ребер. В лемме 1 показано, что все подсистемы уравнений, соответствующие гипердеревьям из $r-1$ и менее ребер, совместны, и описан вид несовместных подсистем уравнений, соответствующих гипердеревьям из $r$ ребер. Алгоритм $A l_{p}$ осуществляет поиск именно таких несовместных подсистем уравнений. В доказательстве теоремы 1 показано, что в случайном гиперграфе $M G(S)$ при условии (4) распределение числа гипердеревьев, соответствующих рассматриваемым несовместным подсистемам из $r$ уравнений, 
сходится к распределению Пуассона со средним значением $\lambda$, приведенным в утверждении теоремы 1. Следовательно, предел суммы вероятностей реализаций случайной системы уравнений $S$, имеющих такие несовместные подсистемы, при $n \rightarrow \infty$ эквивалентен величине $1-e^{-\lambda}$. Учитывая формулу $1-\mathbf{P}_{c}(S) \sim 1-e^{-\lambda}$, следующую из теоремы 1 , убеждаемся, что почти все несовместные реализации случайной системы уравнений $S$ содержат минимальные несовместные подсистемы из $r$ уравнений, описываемые условиями (L1), (L2) леммы 1 . Оценка $\pi_{n} \sim 1$ при условии (4) следует из последнего вывода и определения величины $\pi_{n}$. Из вышеупомянутого результата о предельном пуассоновском распределении числа несовместных подсистем из $r$ уравнений и вида его среднего значения $\lambda$ следует, что при $M / n^{1-1 / r} \rightarrow \infty$ почти все реализации случайной системы уравнений $S$ содержат эти минимальные несовместные подсистемы из $r$ уравнений. Отсюда получаем, что доказанная при условии (4) часть теоремы справедлива и при $M / n^{1-1 / r} \rightarrow \infty$.

Вторая часть утверждения теоремы об отсутствии других несовместных подсистем при дополнительном условии $M=o\left(n^{1-1 /(r+1)}\right)$ следует из того, что в соответствии с теоремами 5, 6 при этом условии почти все реализации случайного гиперграфа $M G(S)$ состоят из гипердеревьев с $r$ или менее ребрами.

Обозначим через $\zeta_{n}$ число решений случайной системы уравнений $S$. В доказательстве следующей леммы применены идеи Г. В. Балакина, В. А. Копытцева, использующиеся при получении аналогичных, но более тонких результатов в случае равновероятной бесповторной выборки неизвестных (см., например, [1], [16]).

Лемма 3. Если $R^{(0)} R^{(1)}>0$, то существует константа $\beta>0$ такая, что $\mathbf{E} \zeta_{n}=o(1) n p u \underline{\lim _{n \rightarrow \infty}} M / n>\beta$.

Доказательство. Обозначим через $V_{n}=V_{n}(B)$ множество векторов размерности $n$ с компонентами, принимающими значения в множестве $B$. Пусть $P(v)$ - вероятность того, что вектор $v=\left(v_{1}, \ldots, v_{n}\right)$ из $V_{n}$ удовлетворяет случайно выбираемой реализации случайного уравнения (3a), $\bar{P}(v)=1-P(v)$. Тогда справедлива оценка

$$
\mathbf{E} \zeta_{n}=\sum_{v \in V_{n}}[P(v)]^{M}
$$

Обозначим через $n_{0}=n_{0}(v)$ число компонент вектора $v$, равных 0 , пусть $n_{1}=n_{1}(v)=n-n_{0}$. Из условия $R^{(0)} R^{(1)}>0$ следует существование наборов 
$\left(i_{0}, j_{0}, a_{0}\right),\left(i_{1}, j_{1}, a_{1}\right)$, удовлетворяющих условию (5), поэтому с помощью формулы (1) при $n_{0}>\frac{n}{2}$ получаем оценку

$$
\begin{gathered}
\bar{P}(v) \geq c_{i_{0}, j_{0}, a_{0}} \bar{P}_{i_{0}, j_{0}, a_{0}}(v) \geq c_{i_{0}, j_{0}, a_{0}} \sum_{\substack{<s_{1}, \ldots, s_{i_{0}}>: \\
v_{s_{1}}=\ldots=v_{s_{0}}=0}} p_{s_{1} \ldots p_{s_{i_{0}}}} \geq \\
\geq c_{i_{0}, j_{0}, a_{0}}\left(\frac{\varepsilon_{1}}{n}\right)^{i_{0}} n_{0}^{i_{0}}>c_{i_{0}, j_{0}, a_{0}}\left(\frac{\varepsilon_{1}}{2}\right)^{i_{0}},
\end{gathered}
$$

где $\bar{P}_{i_{0}, j_{0}, a_{0}}(v)-$ вероятность того, что вектор $v$ не удовлетворяет случайно выбираемой реализации (3) случайного уравнения (3a) при $(i, j, a)=$ $=\left(i_{0}, j_{0}, a_{0}\right), \quad c_{i_{0}, j_{0}, a_{0}}-$ вероятность выбора уравнения с функцией $f_{i_{0}, j_{0}}$ в левой части, величиной $a_{0}$ в правой части, сумма по $<s_{1}, \ldots, s_{i_{0}}>$ является нижней оценкой вероятности $\bar{P}_{i_{0}, j_{0}, a_{0}}(v), n_{0}^{i_{0}}-$ число вариантов выбора $i_{0}$ компонент вектора $v$, равных 0. Следовательно,

$$
P(v)<1-c_{i_{0}, j_{0}, a_{0}}\left(\frac{\varepsilon_{1}}{2}\right)^{i_{0}}=\delta_{0}<1 .
$$

Аналогично при $n_{0} \leq \frac{n}{2}$ убеждаемся в справедливости формулы

$$
\begin{gathered}
\bar{P}(v) \geq c_{i_{1}, j_{1}, a_{1}} \bar{P}_{i_{1}, j_{1}, a_{1}}(v) \geq c_{i_{1}, j_{1}, a_{1}} \sum_{\substack{<s_{1}, \ldots, s_{i_{1}}>: \\
v_{s_{1}} \ldots=v_{s_{i_{1}}}}} p_{s_{1} \ldots p_{s_{i_{1}}} \geq} \geq \\
\geq c_{i_{1}, j_{1}, a_{1}}\left(\frac{\varepsilon_{1}}{n}\right)^{i_{1}} n_{1}^{i_{1}} \geq c_{i_{i_{1}, j_{1}, a_{1}}\left(\frac{\varepsilon_{1}}{2}\right)^{i_{1}},},
\end{gathered}
$$

где $\bar{P}_{i_{1}, j_{1}, a_{1}}(v)-$ вероятность того, что вектор $v$ не удовлетворяет случайно выбираемой реализации (3) случайного уравнения (3a) при $(i, j, a)=$ $=\left(i_{1}, j_{1}, a_{1}\right), \quad c_{i_{1}, j_{1}, a_{1}}-$ вероятность выбора уравнения с функцией $f_{i_{1}, j_{1}}$ в левой части, величиной $a_{1}-$ в правой части, сумма по $<s_{1}, \ldots, s_{i_{1}}>$ является нижней оценкой вероятности $\bar{P}_{i_{1}, j_{1}, a_{1}}(v), n_{1}^{i_{1}}$ - число вариантов выбора $i_{1}$ компонент вектора $v$, равных 1 . Следовательно

$$
P(v) \leq 1-c_{i_{1}, j_{1}, a_{1}}\left(\frac{\varepsilon_{1}}{2}\right)^{i_{1}}=\delta_{1}<1 .
$$


Тогда для любого вектора $v$ из $V_{n}$ выполняется неравенство $P(v) \leq \delta<1$, где $\delta=\max \left\{\delta_{0}, \delta_{1}\right\}<1$. Отсюда следует оценка

$$
\mathbf{E} \zeta_{n} \leq \sum_{0 \leq n_{1} \leq n}\left(\begin{array}{c}
n \\
n_{1}
\end{array}\right) \delta^{M}=2^{n} \delta^{M} .
$$

Поэтому $\mathbf{E} \zeta_{n}=o(1)$ при условии $M-n \beta \rightarrow \infty, \beta=\log _{1 / \delta} 2$.

Возможное совпадение выбираемых неизвестных для уравнений не оказывает влияния на результат, так как в доказательстве используются только уравнения с одинаковыми значениями неизвестных (суммы по $<s_{1}, \ldots, s_{i_{k}}>, k=0,1$ в оценках для $\left.P(v)\right)$. Лемма 3 доказана.

Доказательство теоремы 2. Пусть выполнены условия $R^{(0)} R^{(1)}>0$ и $c^{(0)}+c^{(1)}=0$. Почти все реализации случайного гиперграфа $M G(S)$ при условии $M=o(n)$ состоят из гипердеревьев. Из леммы 2 статьи [25] следует, что при условии $c^{(0)}+c^{(1)}=0$ подсистемы, соответствующие гипердеревьям, совместны. Следовательно, при $M=o(n)$ и $c^{(0)}+c^{(1)}=0$ почти все реализации случайной системы уравнений $S$ совместны независимо от значения величины $R^{(0)} R^{(1)}$. В соответствии с леммой 3 при $M / n \rightarrow \infty, R^{(0)} R^{(1)}>0$ для числа решений $\zeta_{n}$ случайной системы уравнений $S$ справедлива оценка $\mathbf{E} \zeta_{n}=o(1)$, из которой с учетом неравенства $\mathbf{P}\left(\zeta_{n} \geq 1\right) \leq \mathbf{E} \zeta_{n}$ следует, что почти все реализации случайной системы уравнений $S$ несовместны независимо от значения суммы $c^{(0)}+c^{(1)}$. Таким образом, условия $R^{(0)} R^{(1)}>0, c^{(0)}+c^{(1)}=0$ достаточны для наличия у случайной системы уравнений $S$ пороговой функции совместности $n$.

Необходимость условия $\left\{R^{(0)} R^{(1)}>0\right\} \bigcap\left\{c^{(0)}+c^{(1)}=0\right\}$ для того, чтобы случайная система уравнений $S$ имела пороговую функцию совместности $n$, следует из того, что при справедливости отрицания этого условия, имеющего вид $\left\{R^{(0)} R^{(1)}=0\right\} \bigcup\left\{\left\{R^{(0)} R^{(1)}>0\right\} \cap\left\{c^{(0)}+c^{(1)}>0\right\}\right\}$, случайная система уравнений $S$ не имеет пороговой функции совместности, равной $n$. Действительно, если $R^{(0)} R^{(1)}=0$, то в соответствии со следствием 1 случайная система уравнений $S$ не имеет никакой пороговой функции совместности в том числе - вида $n$. Если же $\left\{R^{(0)} R^{(1)}>0, c^{(0)}+c^{(1)}>0\right\}$, то в соответствии с теоремой 1 случайная система уравнений $S$ имеет пороговую функцию совместности, которая не равна $n$. Теорема 2 доказана.

Доказательство теоремы 3. Эквивалентность условий (C2) и (C3) следует из определения величин $R^{(0)}, R^{(1)}$. Покажем эквивалентность условий (C1) и (C2), а также справедливость утверждения о виде пороговых функций совместности. Каждая из величин $R^{(0)} R^{(1)}$ и $c^{(0)}+c^{(1)}$ может быть либо положительна, либо равна нулю. При $R^{(0)} R^{(1)}=0$ случайная система уравнений $S$ имеет решение $\mathbf{0}$ и/или 1 и, следовательно, не имеет пороговой функции со- 
вместности. Рассмотрим случай $R^{(0)} R^{(1)}>0$. Если $c^{(0)}+c^{(1)}=0$, то в соответствии с теоремой 2 случайная система уравнений $S$ имеет пороговую функцию совместности $n$. Если же $c^{(0)}+c^{(1)}>0$, то ввиду условия $R^{(0)} R^{(1)}>0$ найдется $b \in B$ такое, что выполняются соотношения $c^{(b)}>0,1 \leq R^{(b)} \leq m$, и в соответствии с теоремой 1 случайная система уравнений $S$ имеет пороговую функцию совместности $n^{1-1 / r}, 2 \leq r=R^{(b)}+1 \leq m+1$. Таким образом, при $R^{(0)} R^{(1)}=0$ случайная система уравнений $S$ не имеет пороговой функции совместности, а при $R^{(0)} R^{(1)}>0$ случайная система уравнений $S$ имеет пороговую функцию совместности указанного в утверждении теоремы 3 вида. Теорема 3 доказана.

Доказательство следствия 2. Доказательство теоремы 1 для бесповторной выборки неизвестных в уравнениях систем аналогично доказательству следствия 3 , оно использует лемму 2 для случайной системы уравнений $S$. Пусть свойство $Q$ заключается в отсутствии в реализациях $S$ уравнений с совпадающими индексами неизвестных. В соответствии с теоремой 5 при $M=o(n)$ почти все реализации $M G(S)$ не имеют особых ребер. Из этого с учетом установленной в п. 2 связи между случайной системой уравнений $S$ и соответствующим ей случайным гиперграфом следует, что при $M=o(n)$ почти все реализации $M G(S)$ не имеют уравнений с совпадающими индексами неизвестных. Таким образом, условие леммы 2 выполнено. Выберем свойство $Q^{*}$, заключающееся в совместности реализации случайной системы уравнений $S$. Из леммы 2 и определений свойств $Q^{*}$ и $Q$ при $M=o(n)$ следует существование и совпадение следующих пределов

$$
\lim _{n \rightarrow \infty} \mathbf{P}_{c}(S)=\lim _{n \rightarrow \infty} \mathbf{P}\left(Q^{*}\right)=\lim _{n \rightarrow \infty} \mathbf{P}\left(Q^{*} \mid Q\right)=\lim _{n \rightarrow \infty} \mathbf{P}_{c}(S \mid Q) .
$$

В соответствии с теоремой 1 указанные пределы равны 1,0 и $e^{-\lambda}$ при $M=o\left(n^{1-1 / r}\right), M / n^{1-1 / r} \rightarrow \infty$ и при условии (4) соответственно (параметр $\lambda$ определен в условии теоремы 1). Отсюда следует справедливость утверждений теоремы 1 для случайной системы уравнений $S$ с бесповторной выборкой неизвестных в уравнениях.

Доказательство теоремы 4 использует теоремы 1, 5, 6 и лемму 1. Справедливость указанных теорем для случайной системы уравнений $S$ с бесповторной выборкой неизвестных показана выше в доказательстве и в следствии 3. Утверждение леммы 1 сформулировано для гипердеревьев, которые не могут иметь особых ребер. Таким образом, утверждение теоремы 4 справедливо и для случайной системы уравнений $S$ с бесповторной выборкой неизвестных.

Утверждение следствия 1 вытекает из определения величины $R^{(b)}$, $b \in B$. Так как понятие $R^{(b)}$ введено для бесповторной выборки неизвестных в уравнении (3), то следствие 1 справедливо и для случайной системы уравнений $S$ с бесповторной выборкой неизвестных. 
Доказательство леммы 3 справедливо и для случайной системы уравнений $S$ с бесповторной выборкой неизвестных. При этом оценки вероятности $\bar{P}(v)$ сохраняются: в суммах по $<s_{1}, \ldots, s_{i_{k}}>, k=0,1$, перебор индексов суммирования осуществляется по схеме без возвращения, а число $n_{k}^{i_{k}}$ вариантов выбора $i_{k}$ компонент вектора $v$, равных $k$, заменяется на $\left(n_{k}\right)_{i_{k}}$.

Выше в доказательстве со ссылкой на теорему 5 показано, что при $M=o(n)$ почти все реализации случайной системы уравнений $S$ не имеют уравнений с совпадающими неизвестными. Поэтому утверждения теорем 2 , 3 в случае $M=o(n)$ сохраняются и для случайной системы уравнений $S$ с бесповторной выборкой неизвестных. Утверждения этих теорем в случаях, когда условие $M=o(n)$ не выполняется, справедливы и при бесповторной выборке неизвестных, так как их доказательства основаны на лемме 3, доказанной выше для такой выборки неизвестных.

\section{4. Трудоемкость алгоритмов}

Оценим трудоемкость алгоритма $A_{k l}$. Поиск неизвестных, определяющихся из конечного дискретного уравнения вида (3), можно осуществить следующим образом. Неизвестное $x_{s_{k}}$ однозначно определяется из уравнения (3) и равно $b \in B$ тогда и только тогда, когда подфункция $g_{s_{k}}^{b}\left(y_{1}, \ldots, y_{i}\right)$ функции $g\left(y_{1}, \ldots, y_{i}\right)=f_{i, j}\left(y_{1}, \ldots, y_{i}\right)$ удовлетворяет тождеству $g_{s_{k}}^{b}\left(y_{1}, \ldots, y_{i}\right) \equiv a$, где $1 \leq s_{k} \leq i, a \in A$ (т. е. переменные с номерами из $\{1, \ldots, i\} \backslash\left\{s_{k}\right\}$ несущественно входят в функцию относительно фиксации неизвестного $x_{s_{k}}$ значением $b$ ). Для проверки этого условия достаточно перебрать все $2^{i-1}$ возможных значений оставшихся $i-1$ переменных подфункции $g_{s_{k}}^{b}\left(y_{1}, \ldots, y_{i}\right)$ и сформировать множество ее значений. Оно равно $\{a\}$ тогда и только тогда, когда неизвестное $x_{s_{k}}$ однозначно определяется из уравнения (3) и равно $b$.

Перейдем к задаче определения ранга $R^{(b)}$ несовместных фиксаций значением $b$ неизвестных уравнения случайной системы $S$ и перечисления множеств с несовместной $R^{(b)}$-фиксацией значением $b$ неизвестных в допустимых уравнениях вида (3) (для них $c_{i, j, a}>0$ ). Зафиксируем возможное значение $t$ ранга $R^{(b)}, t \in\{1, \ldots, m\}$. Для каждого множества $\left\{k_{1}, \ldots, k_{t}\right\} \subseteq\{1, \ldots, i\}$, состоящего из $t$ номеров индексов неизвестных уравнения (3), перебираются все $2^{i-t}$ возможных значений $i-t$ переменных подфункции $g_{k_{1}, \ldots, k_{t}}^{b, \ldots, b}\left(y_{1}, \ldots, y_{i}\right)$ функции $g=f_{i, j}$ в уравнении (3) и формируется множество ее значений. Если элемент $a \in A$ не принадлежит множеству зна- 
чений подфункции $g_{k_{1}, \ldots, k_{t}}^{b, \ldots, b}\left(y_{1}, \ldots, y_{i}\right)$, то множество $\left\{k_{1}, \ldots, k_{t}\right\}$ является несовместной $t$-фиксацией значением $b$ неизвестных уравнения вида (3) и $R^{(b)} \leq t$. Перебор всех $\left(\begin{array}{l}i \\ t\end{array}\right)$ возможных вариантов несовместных $t$ фиксаций значением $b$ неизвестных уравнения вида (3) позволяет сделать вывод об отсутствии искомых несовместных $t$-фиксаций либо об их наличии, числе и конкретных вариантах. Если возможные значения ранга $R^{(b)}$ перебираются в возрастающем порядке и найдена первая несовместная $t$ фиксация значением $b$ уравнения вида $(3)$, то $R^{(b)}=t$. Если перебраны значения $1, \ldots, m$ и не найдена ни одна несовместная фиксация значением $b$ неизвестных уравнения вида (3), то $R^{(b)}=0$.

Из вышеизложенных рассуждений, описания алгоритма $A_{k l}$, ограниченности числа перебираемых уравнений и величин $|B|,|A|$ и $m$ следует, что трудоемкость алгоритма $A_{k l}$ не больше некоторой ограниченной величины.

Определим среднюю и максимальную трудоемкость $T_{\mathrm{cp}}\left(A l_{p}\right), T_{\mathrm{cp}}(\boldsymbol{U})$ и $T_{\max }\left(A l_{p}\right), T_{\max }(\boldsymbol{U})$ алгоритма $A l_{p}$ и алгоритма $\boldsymbol{U}$ полного перебора решений как среднее и максимальное значения случайных величин, равных трудоемкости решения задачи распознавания несовместности случайно выбираемой реализации случайной системы уравнений $S$ соответствующим алгоритмом.

Если $R^{(0)} R^{(1)}=0$, то алгоритм $A l_{p}$ на шаге 0 (фактически - алгоритм $A_{k l}$ на шаге 4) завершает работу с выводом о том, что случайная система уравнений $S$ заведомо совместна. В этом случае $T_{\max }\left(A l_{p}\right)=O(1)$, $T_{\mathrm{cp}}\left(A l_{p}\right)=O(1)$.

Пусть $R^{(0)} R^{(1)}>0$. На шаге 1 алгоритма $A l_{p}$ запись информации о значениях определившихся неизвестных требует не более некоторого ограниченного числа $k_{1}$ операций. При $r=2$ на шаге 2 проверка наличия противоречия между определившимся значением неизвестного из очередного уравнения с полученным ранее значением этого неизвестного требует не более некоторого ограниченного числа $k_{2}$ операций. При $r>2$ на шаге 3 перебор несовместных $(r-1)$-фиксаций значением $b$ в каждом уравнении требует не более некоторого ограниченного числа $k_{3}$ операций. Число уравнений в каждой системе равно $M$, число неизвестных в каждом уравнении не более $m$, поэтому при $R^{(0)} R^{(1)}>0$ справедливы оценки $T_{\max }\left(A l_{p}\right)=$ $=O\left(M\left(m k_{1}+m k_{2}+k_{3}\right)\right)=O(M), T_{\mathrm{cp}}\left(A l_{p}\right)=O(M)$.

Пусть алгоритм $\boldsymbol{U}$ тратит не менее $k_{0} \geq 1$ операций на проверку совместности одного уравнения при подстановке одного решения. Так как случайная система уравнений $S$ при любом числе уравнений имеет совместную реализацию, то $T_{\max }(\boldsymbol{U})=k_{0} M|B|^{n}$. Вероятность случайного выбора совместной реализации случайной системы уравнений $S$ равна $\mathbf{P}_{c}(S)$. Следовательно, 
при $\lim _{n \rightarrow \infty} \mathbf{P}_{c}(S) \geq \rho>0 \quad$ справедлива $\quad$ оценка $\quad T_{\mathrm{cp}}(\boldsymbol{U})=O\left(k_{1} M|B|^{n} \mathbf{P}_{c}(S)\right)=$ $=O\left(M|B|^{n}\right)$.

В работе [3] метод решения систем уравнений называется элементарHblм, если для нахождения одного решения потребуется затратить $O\left(T_{0}(M)\right)$ элементарных операций, где $T_{0}(M)$ - трудоемкость записи системы в память ЭВМ. Трудоемкость такого метода сравнима с простотой маркировки (просмотром) массива исходных данных. По аналогии алгоритм распознавания несовместности реализаций случайной системы уравнений назовем элементарным, если для распознавания несовместности реализаций случайной системы уравнений потребуется затратить $O\left(T_{0}(M)\right)$ элементарных операций. Легко видеть, что алгоритм $A l_{p}$ является элементарным.

\section{5. Примеры}

Рассмотрим случайную систему уравнений $S$, уравнения (3) которой имеют вид

$$
f_{t d, j}\left(x_{s_{1}}, \ldots, x_{s_{d t}}\right)=\sum_{k=1}^{t} f_{d, j}\left(x_{s_{d(k-1)+1}}, \ldots, x_{s_{d(k-1)+d}}\right)=a,
$$

$t=1, \ldots,\lfloor m / d\rfloor, f_{t d, j}$ - псевдобулева функция, равная сумме в поле действительных чисел $t$ булевых функций $f_{d, j}$ из $\Phi_{d}, d \geq 1$, правая часть $a$ уравнения (8) принимает целые значения $0,1, \ldots, t$. В статье [25] для случайной равновероятной выборки без повторения рассмотрены примеры, в которых функции в (8) имеют вид $f_{1,1}\left(y_{1}\right)=y_{1}, d=1$ (в [29] рассмотрен случай $c_{m}=1$, более общий случай $|B| \geq 2$ рассмотрен в статьях [5], [24]); $f_{d, 2}\left(y_{1}, \ldots, y_{d}\right)=y_{1}\left(y_{2} \oplus \ldots \oplus y_{d}\right), \quad d \geq 3 ; \quad f_{d, 3}\left(y_{1}, \ldots, y_{d}\right)=\bar{y}_{1}\left(y_{2} \oplus \ldots \oplus y_{d}\right), \quad d \geq 3 ;$ $f_{d, 4}\left(y_{1}, \ldots, y_{d}\right)=y_{1} \vee \ldots \vee y_{d}, d \geq 2$ (для $f_{d, 4}$ случай $c_{2}=1, m=d=2$ изучался $\Gamma$. В. Балакиным в [1], случай $d=2, m=t d, c_{m}=1-$ В. А. Копытцевым); примеры с функциями $f_{d, 2}, f_{d, 3}, f_{d, 4}$ при $m=d, c_{m}=1$ рассмотрены в [23]. Случайная система уравнений $S$ с уравнениями вида (8) для приведенных примеров имеет такие же пороговые функции совместности, как и при случайной равновероятной выборке без повторения в статье [25]. Для предельной вероятности совместности случайной системы уравнений $S$, имеющей пороговую функцию совместности, при условии (4), как и в работе [25], справедлива формула $\mathbf{P}_{c}(S) \sim e^{-\lambda}$. Параметр $\lambda$ в статье [25] равен $c^{r} c^{(b, r-1)}\left(c^{(b)}\right)^{r-1}$, в условиях данной работы $-c^{r} c^{(b, r-1)}\left(\sigma^{2} c^{(b)}\right)^{r-1}$, а величины $c^{(b, r-1)}, c^{(b)}$ при $b=0,1$ в статье [25] и в данной работе одинаковы.

Если функция $f_{d, j}$ в (8) не имеет однозначно определяющихся неизвестных $\left(c^{(0)}+c^{(1)}=0\right)$ и $R^{(0)} R^{(1)}>0$, то случайная система уравнений $S$ имеет порого- 
вую функцию совместности $n$. Так, например, если функции в уравнениях случайной системы $S$ булевы и являются элементарными симметрическими многочленами $\sum_{1 \leq i_{1}<i_{2}<\ldots<i_{k} \leq m} y_{i_{1}} y_{i_{2}} \ldots y_{i_{k}}$, где $k$ фиксировано, $2 \leq k \leq m-1$, $m \geq 3$, то при $R^{(0)} R^{(1)}>0$ случайная система уравнений $S$ имеет пороговую функцию совместности $n$.

Пример 1. Допустимые уравнения имеют вид

$$
f_{3,6}\left(x_{s_{1}}, x_{s_{2}}, x_{s_{3}}\right)=x_{s_{1}} x_{s_{2}} x_{s_{3}}=a, B=\{2,3\}, a \in A=\{8,12,18,27\},
$$

операция умножения рассматривается в множестве целых чисел.

При $a=12$ или $a=18$ такие уравнения не имеют частично определяющихся неизвестных, а при $a=8$ и $a=27$ все неизвестные однозначно определяются и равны 2 и 3 соответственно, т. е. $c^{(2)}=c^{(3,1)}=c_{3,6,8}, c^{(3)}=c^{(2,1)}=c_{3,6,27}$. Кроме того, $c^{(2,2)}=c^{(3,3)}=c_{3,6,18}, c^{(2,3)}=c^{(3,2)}=c_{3,6,12}$. Поэтому в соответствии с теоремами 1-3 случайная система уравнений $S$ имеет пороговую функцию совместности $\sqrt{n}$ при $c_{3,6,8} c_{3,6,27}>0, n^{2 / 3}$ при $c_{3,6,27}=0, c_{3,6,8} c_{3,6,18}>0$ и при $c_{3,6,8}=0, c_{3,6,12} c_{3,6,27}>0, n^{3 / 4}$ при $c_{3,6,27}=c_{3,6,18}=0, c_{3,6,8} c_{3,6,12}>0$ и при $c_{3,6,8}=c_{3,6,12}=0, c_{3,6,18} c_{3,6,27}>0, n$ при $c_{3,6,8}+c_{3,6,27}=0$. В соответствии со следствием 1 случайная система уравнений $S$ имеет решение $\mathbf{b}$ при $b=2$ и $b=3$, если правые части всех уравнений равны 8 и 27 соответственно.

Пример 2. Допустимые уравнения имеют вид

$$
f_{3,7}\left(x_{s_{1}}, x_{s_{2}}, x_{s_{3}}\right)=x_{s_{1}} x_{s_{2}}+x_{s_{1}} x_{s_{3}}+x_{s_{2}} x_{s_{3}}=a, a \in A,
$$

операции умножения и сложения рассматриваются в множестве целых чисел.

Пусть $B=\{1,2\}, A=\{3,5,8,12\}$. Только при $a=3$ и $a=12$ все неизвестные однозначно определяются и равны 1 и 2 соответственно, т. е. $c^{(1)}=c^{(2,1)}=c_{3,7,3}, \quad c^{(2)}=c^{(1,1)}=c_{3,7,12} . \quad$ Кроме того, $\quad c^{(1,2)}=c^{(2,3)}=c_{3,7,8}$, $c^{(1,3)}=c^{(2,2)}=c_{3,7,5}$. Поэтому в соответствии с теоремами 1-3 случайная система уравнений $S$ имеет пороговую функцию совместности $\sqrt{n}$ при $c_{3,7,3} c_{3,7,12}>0, n^{2 / 3}$ при $c_{3,7,12}=0, c_{3,7,3} c_{3,7,8}>0$ и при $c_{3,7,3}=0, c_{3,7,12} c_{3,7,5}>0, n^{3 / 4}$ при $c_{3,7,12}=c_{3,7,8}=0, c_{3,7,3} c_{3,7,5}>0$ и при $c_{3,7,3}=c_{3,7,5}=0, c_{3,7,12} c_{3,7,8}>0, n$ при $c_{3,7,3}+c_{3,7,12}=0$. В соответствии со следствием 1 случайная система уравнений $S$ имеет решение $\mathbf{b}$ при $b=1$ и $b=2$, если правые части всех уравнений равны 3 и 12 соответственно.

Если же $B=\{-1,1\}, A=\{-1,3\}$, то уравнения с однозначно определяющимися неизвестными отсутствуют. В соответствии с теоремой 2 и следствием 1 случайная система уравнений $S$ имеет пороговую функцию совместности $n$ при $c_{3,7,3}<1$ и имеет решения $\mathbf{b}$ для $b=1$ и $b=-1$ при $c_{3,7,3}=1$.

Пример 3. Допустимые уравнения имеют вид $f_{3,7}\left(x_{s_{1}}, x_{s_{2}}, x_{s_{3}}, x_{s_{4}}\right)=x_{s_{1}} x_{s_{2}} x_{s_{3}}+x_{s_{1}} x_{s_{2}} x_{s_{4}}+x_{s_{1}} x_{s_{3}} x_{s_{4}}+x_{s_{2}} x_{s_{3}} x_{s_{4}}=a, a \in A$, операции умножения и сложения рассматриваются в множестве целых чисел.

Рассмотрим случай $B=\{1,2\}, A=\{4,9,12,20,32\}$. Только при $a=4$ и $a=32$ все неизвестные однозначно определяются и равны 1 и 2 соответст- 
венно, т. е. $\quad c^{(1)}=c^{(2,1)}=c_{4,8,4}, \quad c^{(2)}=c^{(1,1)}=c_{4,8,32}$. С помощью теорем $1-3$ и следствия 1 можно выписать необходимые и достаточные условия наличия у случайной системы уравнений $S$ пороговых функций совместности вида $n, n^{1-1 / r}, r=2,3,4,5$ и решения $\mathbf{b}$ при $b=1$ и $b=2$, а также предельные оценки вероятности совместности случайной системы уравнений $S$ при условии (4).

Пусть теперь $B=\{-1,1\}, A=\{-4,-2,2,4\}$. Только при $a=4$ и $a=-4$ все неизвестные однозначно определяются из уравнения и равны 1 и -1 соответственно, т. е. $\quad c^{(1)}=c^{(-1,1)}=c_{4,8,4}, \quad c^{(-1)}=c^{(1,1)}=c_{4,8,-4} . \quad$ С помощью теорем 1-3 и следствия 1 несложно выписать необходимые и достаточные условия наличия у случайной системы уравнений $S$ пороговых функций совместности вида $n, \sqrt{n}, n^{2 / 3}, n^{4 / 5}$ и решения $\mathbf{b}$ для $b=1$ и $b=-1$, а также предельные оценки вероятности совместности случайной системы уравнений $S$ при условии (4). Интересно отметить, что в списке пороговых функций совместности отсутствует функция $n^{3 / 4}$, так как нет допустимых несовместных подсистем уравнений из 4 уравнений, удовлетворяющих условиям (L1), (L2) леммы 1.

Пример 4. Функции в уравнениях случайной системы $S$ являются элементарными симметрическими многочленами с булевыми переменными и операцией сложения в кольце целых чисел $\sum_{1 \leq i_{1}<i_{2}<\ldots<i_{k} \leq m} y_{i_{1}} y_{i_{2}} \ldots y_{i_{k}}$, где $k$ фиксировано, $2 \leq k \leq m, m \geq 3$, а правые части уравнений могут принимать значения 0 и $\left(\begin{array}{l}m \\ k\end{array}\right)$ (минимальное и максимальное возможные значения функции). Значения функции в уравнениях в зависимости от числа переменных в функции $m=3,4,5$, параметра $k=2, \ldots, m$ и веса $v=0,1, \ldots, m$ вектора решения (числа единиц в решении) приведены в таблице 1.

Таблица 1

\begin{tabular}{|c|c|c|c|c|c|c|c|c|c|}
\hline \multirow{2}{*}{$v$} & \multicolumn{3}{|c|}{$m=3$} & \multicolumn{3}{c|}{$m=4$} & \multicolumn{4}{c|}{$m=5$} \\
\cline { 2 - 11 } & $k=2$ & $k=3$ & $k=2$ & $k=3$ & $k=4$ & $k=2$ & $k=3$ & $k=4$ & $k=5$ \\
\hline 0 & 0 & 0 & 0 & 0 & 0 & 0 & 0 & 0 & 0 \\
\hline 1 & 0 & 0 & 0 & 0 & 0 & 0 & 0 & 0 & 0 \\
\hline 2 & 1 & 0 & 1 & 0 & 0 & 1 & 0 & 0 & 0 \\
\hline 3 & 3 & 1 & 3 & 1 & 0 & 3 & 1 & 0 & 0 \\
\hline 4 & - & - & 6 & 3 & 1 & 6 & 3 & 1 & 0 \\
\hline 5 & - & - & - & - & - & 10 & 10 & 5 & 1 \\
\hline
\end{tabular}

Уравнения имеют однозначно определяющиеся неизвестные (все $m$ неизвестных равны 1) только в случае, когда правая часть уравнений принимает максимально возможное значение функции. Несовместные подсистемы, описываемые условиями (L1), (L2) леммы 1 , состоят из $k+1$ уравнения, 
первые $k$ уравнений имеют в правой части $\left(\begin{array}{c}m \\ k\end{array}\right),(k+1)$-е уравнение - 0. Несовместных подсистем из $k$ и менее уравнений нет. Например, при $m=5$, $k=2, \ldots, 5$ из уравнения с 0 в правой части следует, что вес $v$ вектора решения уравнения удовлетворяет формуле $0 \leq v \leq k-1$. Следовательно, решение уравнения имеет не более $k-1$ неизвестных, равных 1 . Так как $k$ неизвестных этого уравнения входят в другие уравнения, в которых они однозначно определяются и равны 1 , то приведенная подсистема из $k+1$ уравнения несовместна. В соответствии с теоремой 1 пороговая функция совместности равна $n^{k /(k+1)}$, т. е. $n^{2 / 3}, n^{3 / 4}, n^{2 / 3}, n^{3 / 4}, n^{4 / 5}, n^{2 / 3}, n^{3 / 4}, n^{4 / 5}, n^{5 / 6}$ при $m=3, k=2,3 ; m=4, k=2,3,4 ; m=5, k=2, \ldots, 5$.

Автор выражает благодарность рецензентам Г. В. Балакину, А. М. Зубкову за замечания, позволившие улучшить содержание работы.

\section{Список литературы}

1. Балакин Г. В. Графы систем двучленных уравнений с булевыми неизвестными. - Теория вероятностей и ее применения, 1995, т. 40, вып. 2 , c. $241-259$.

2. Балакин Г. В. О вероятностном подходе к решению систем уравнений с целочисленными неизвестными. - Дискрет. матем., 1995, т. 7, вып. 1, c. $88-98$.

3. Балакин Г. В. Эффективно решаемые классы систем булевых уравнений. - Обозрение прикладной и промышленной математики, 1995, т. 2 , в. 3, с. 494-501.

4. Балакин Г. В. Введение в теорию случайных систем уравнений. - Труды по дискретной математике. Т. 1. - М.: ТВП, 1997, с. 1-18.

5. Балакин Г. В. Системы случайных уравнений над конечным полем. Труды по дискретной математике. Т. 2. - М.: ТВП, 1998, с. 21-28.

6. Балакин Г. В. Системы случайных булевых уравнений со случайным выбором неизвестных в каждом уравнении. - Труды по дискретной математике. - М.: ФИЗМАТЛИТ, 2000, т. 3, с. 21-28.

7. Балакин Г. В., Бачурин С. А. Оценка параметров метода последовательного подбора неизвестных. - Труды по дискретной математике. Т. 6. М.: Физматлит, 2002, с. 7-13.

8. Балакин Г. В., Колчин В. Ф., Хохлов В. И. Гиперциклы в случайном гиперграфе. - Дискретн. матем., 1991, т. 3, вып. 3, с. 102-108.

9. Зыков А. А. Гиперграфы. - Успехи матем. наук, 1974, т. 29, вып. 6, с. 89-154.

10. Колчин В. Ф. Системы случайных уравнений. - М.: МИЭМ, 1988. 
11. Колчин В.Ф. Вероятность совместности одной системы случайных уравнений специального вида. - Труды по дискретной математике. Т. 3. - М.: Физматлит, 2000, с. 139-146.

12. Колчин В. Ф. Случайные графы. - М.: Физматлит, 2000.

13. Колчин В. Ф., Хохлов В. И. О числе циклов в случайном неравновероятном графе. - Дискретн. матем., 1990, т. 2, вып. 3, с. 137-145.

14. Колчин В. Ф., Хохлов В. И. Пороговый эффект для систем случайных уравнений специального вида. - Дискретн. матем., 1995, т. 7, вып. 4, с. 29-39.

15. Копытчев B. A. О распределении числа решений случайных заведомо совместных систем уравнений. - Теория вероятностей и ее применения, 1995, т. 40, вып. 2, с. 430-437.

16. Копытцев В. А. О некоторых результатах, связанных с анализом систем случайных уравнений. - Вестник ИКСИ. Серия «К». Спец. вып. - М., 2003, с. 71-75.

17. Никонов В. Г., Никонов Н. В. Запреты $k$-значных функций и их связь с проблемой разрешимости систем уравнений специального вида. Вестник РУДН, серия Прикл. матем. и компьют. матем., 2003, т. 2, вып. 1, c. 79-93.

18. Сачков В. Н. Вероятностные методы в комбинаторном анализе. - М.: Наука, 1978.

19. Сачков B. Н. Случайные неравновероятные покрытия и функциональные уравнения. - Труды по дискретной математике. Т. 5. - М.: Физматлит, 2002, c. 205-218.

20. Сачков В. Н. Введение в комбинаторные методы дискретной математики. - Изд. 2-е. - М.: МЦНМО, 2004.

21. Харари Ф. Теория графов. Изд. 3-е. - М.: КомКнига, 2006.

22. Шаповалов А. В. О числе строго сбалансированных подграфов случайных однородных гиперграфов. - Дискретн. матем., 1993, т. 5, вып. 4, с. $133-$ 144.

23. Шаповалов А. В. Вероятность совместности случайных систем булевых уравнений. - Дискретн. матем., 1995, т. 7, вып. 2, с. 146-159.

24. Шаповалов А. В. Пороговые функции совместности случайных систем уравнений. - Труды по дискретной математике. Т. 9. - М.: Гелиос APB, 2006, с. 377-400.

25. Шаповалов А. В. Совместность и алгоритм распознавания несовместности реализаций случайных систем дискретных уравнений с двузначными неизвестными. - Дискретн. матем., 2008, т. 20, вып. 3, с. 28-39.

26. Шаповалов A. В. Характеристики случайных систем линейных уравнений над конечным полем. - Дискретн. матем., 2008, т. 20, вып. 4, c. $136-146$. 
27. Шаповалов А. В. Предельные характеристики случайных систем линейных уравнений над кольцом вычетов. - Обозрение прикладной и промышленной математики, 2008, т. 15, вып. 6, с. 1003-1018.

28. Шаповалов А. В. Характеристики случайных систем дискретных уравнений при неравновероятной выборке неизвестных. - Математические вопросы криптографии, 2010, т. 1, вып. 3, с. 93-117.

29. Balakin $G$. $V$. On the number of solutions of systems of pseudo-boolean random equations. - Вероятностн. методы дискретн. матем. / Труды 3-й Петрозаводской конференции. - M./Utrecht: TBП/VSP, 1993, с. 71-98.

30. Bollobas B. Random graphs. — London etc.: Academic Press, 1985.

31. Creignou N., Daude H. Smooth and sharp thresholds for random $k$-XORCNF-formulas satisfiability. - Theoret. Informatics and Appl., 2003, v. 37, p. $127-147$.

32. Creignou N., Daude H., Dubois $O$. Approximating the satisfiability threshold for random $k$-XOR-formulas. - Comb., Probab. and Comp., 2003, v. 12, no. 2, p. 113-126.

33. Erdös P., Rényi A. On the evolution of random graphs. - Publ. Math. Inst. Hung. Acad. Sci., Ser. A, 1960, v. 5, p. 17-61.

34. Shapovalov A. V. Characteristics of random systems of Boolean equations with non-regular left-hand side. - Probab. Meth. in Discr. Math. Proc. 5-th Int. Petrozavodsk Conf. VSP.: Utrecht, Boston, Köln, Tokyo, the Netherlands, 2002, p. 345-350. 